Meta

Journal des tradlucteurs

Translators' Journal

\title{
Le sigle : aperçu linguistique
}

\section{Daniel Gehenot}

Volume 20, numéro 4, décembre 1975

URI : https://id.erudit.org/iderudit/002836ar

DOI : https://doi.org/10.7202/002836ar

Aller au sommaire du numéro

Éditeur(s)

Les Presses de l'Université de Montréal

ISSN

0026-0452 (imprimé)

1492-1421 (numérique)

Découvrir la revue

Citer cet article

Gehenot, D. (1975). Le sigle : aperçu linguistique. Meta, 20(4), 271-307.

https://doi.org/10.7202/002836ar d'utilisation que vous pouvez consulter en ligne.

https://apropos.erudit.org/fr/usagers/politique-dutilisation/ 


\section{Le sigle: apercu linguistique*}

\section{GÉNÉRALITÉS}

La vie actuelle coule à un rythme de plus en plus rapide de sorte que, parfois, elle n'accorde plus le temps matériel pour écrire ou même prononcer entièrement certains mots ou certains groupes de mots, ce qui oblige souvent l'écrivain ou l'orateur à se contenter, pour exprimer sa pensée, d'abréviations ou de signes qui se composent de lettres initiales, connues des techniciens, mais ignorées du grand public, pour lequel elles constituent très souvent de vraies énigmes.

Chaque jour voit l'apparition de nouveaux sigles dont les auteurs n'ont pas toujours la délicatesse de donner, au moins une fois, la signification. "Il y aurait un essai à écrire sur le mauvais usage des sigles. Il y en a trop. Ils nous inondent et nous obsèdent en compliquant notre existence. L'encyclopédie Quid de 1972 leur consacre six pages d'une énumération bien tassée où voisinent le «Bic 》 (Bataillon d'infanterie coloniale ou... Bénéfices industriels et commerciaux!) et le « Bird », qui n'est pas un oiseau des îles Britanniques, mais la Banque internationale pour la reconstruction et le développement; le B.C. qui peut être selon les cas le «British Council» ou le «Before Christus» des livres d'histoire anglais. Et, il faut se garder de prendre V.I.P. (very important person) pour V.S.O.P., qui est le "Very Superior Old Pale» (très vieil alcool supérieur) 》 Le Soir, 16-17 avril 1972).

La brachigraphie ${ }^{1}$ est de mode. (Abréviations usuelles, $5^{\mathrm{e}}$ édition, Bruxelles, Sénat, Service d'étude et de documentation, 1958). Elle se glisse partout, nous assaille, nous étouffe.

* Introduction à un glossaire de sigles politiques et économiques en usage en Espagne et dans certains pays d'Amérique de langue espagnole (Argentine, Bolivie, Mexique, Venezuela). Résumé de l'Introduction du travail de fin d'étude présenté en langue espagnole à l'École supérieure de traduction et d'interprétariat de la ville de Bruxelles (Institut d'enseignement supérieur Lucien Cooremans).

1. Brachigraphie (du grec: brakhus, bref, et graphein, écrire). C'est l'art d'abréger les mots, en les représentant conventionnellement par une seule lettre ou par un groupe de lettres, selon une des modalités suivantes :

a) Ellipse totale de toutes les lettres qui suivent la première lettre (le cas le plus fréquent). - Maintien de la lettre initiale (appelée «sigle ») et suppression de toutes les autres lettres. Exemple : a J.O. ", au lieu de " s'il-vous-plaît ».

En aucun cas, l'abréviation ne doit se faire par suppression (aphérèse) de la lettre ou de la syllabe initiale. 
« Être compris par ceux à qui l'on s'adresse est une règle d'or - et, sur ce point, la grammaire a l'honneur de s'identifier avec la charité - que je rappellerai par exemple aux auteurs du Missel dominical de l'Assemblée (Brepols, 1972), ouvrage destiné aux fidèles ordinaires et non aux théologiens. À la suite de chaque messe, il recommande à ces fidèles ordinaires de lire dans la Bible des passages de $\mathbf{P}$, de $\mathrm{R}$, de $\mathrm{S}$, de Jo, de Dn. Qui identifiera du premier coup les livres dont il s'agit? Ces abréviations ne sont pas traditionnelles; soit, il faut bien changer cela aussi. Mais pourquoi les choisir aussi rudes? Et pourquoi ne pas en donner quelque part une liste avec la traduction en clair? $\gg$ (La Libre Belgique Façons de parler : les abréviations, 19/02/1973, par André GOSSE, professeur à l'Université de Louvain).

"We live in an era of abbreviations, or acronyms, a literary disease for which the only cure is extirpation. Such a course is not likely and therefore the only relief is diagnosis without cure. Such a diagnosis has been attempted there " (Buttress, World List of Abbreviations, 1966.

«Acronym agglomeration is an affliction of the age, and there are acronym addicts who, in their weakness, find it impossible to resist them 》 (Moser, SpaceAge Acronyms, Washington/New-York, 1969, Foreword).

Les sigles constituent donc un des aspects de notre vie trépidante, mouvementée, qui a conduit au sacrifice de la qualité par la quantité et la rapidité. Pour transmettre toutes les nouvelles du jour, un journal doit abréger tout ce qui peut l'être, sinon la place lui manquerait très rapidement.

D'autre part, la profusion de comités, organisations, associations, commissions, agences, etc., chacun accompagné de son sigle, conduit à une mauvaise compréhension, ou plus souvent au manque total de compréhension, des divers organismes cités. Ainsi pour comprendre un grand nombre d'articles, la connaissance des sigles est-elle primordiale.

Voici un exemple tiré d'une revue française le Nouvel Observateur $\left(\mathrm{n}^{\circ} 364\right.$, 1-7 novembre 1971, page 25) qui semble assez éloquent: "C'est pourquoi le gouvernement, sensible aux pressions privées, a préféré la solution Franlab : celle-ci doit se chercher d'autres partenaires et devenir le noyau d'un grand groupe privé ; un regroupement avec d'autres centres publics d'informatique (ceux d'E.D.F., du C.N.R.S., de l'Université, etc.) qui est interdit. Car il ne faut surtout pas - écrit le syndicat C.F.D.T. du C.E.A. - que les gros intérêts privés soient lésés au profit de l'intérêt public...»

b) Ellipse partielle. - Maintien de plusieurs lettres initiales consćcutives et suppression (apocope) des lettres terminales. Exemple : "Etym. ", au lieu de "Etymologie»; "Sci. ", au lieu de "Sciences".

c) Syncope. - Maintien de la lettre initiale, ainsi que la lettre ou des lettres terminales, et suppression de toutes les lettres inérieures. Exemple : "Fco", au lieu de "Franco»; "Mme ", au lieu de "Madame ".

d) Contraction. - Maintien de la lettre initiale, en la faisant suivre de lettres choisies arbitrairement dans le mot lui-même et consacrées par l'usage. Exemple : "Cdt », au lieu de "Commandant "; «tjs », au lieu de «toujours".

(Baudry, Hubert: Dictionnaire d'abréviations françaises et étrangères, techniques et zisuelles, Editions de Montligeon, La Chapelle-Montligeon, 1951, page 9, p. 1.) 
Ce paragraphe n'a aucun sens si nous ignorons que le sigle E.D.F. signifie Électricité de France; C.N.R.S., Centre national de la recherche scientifique; C.E.A., Commissariat à l'énergie atomique ; etc.

Enfin, ces quelques lignes tirées de la revue vénézuélienne Semana (Caracas, Ano III, $\mathrm{n}^{\circ} 135, \mathrm{p} .12$ ) : "La mayoría de los dirigentes obreros de URD están en desacuerdo con la división de la CTV, pero la verdad es que si el MEP abandona la Confederación, para URD es muy difícil permanecer en la CTV con COPEL y $A D \ldots$...

Dans le Monde du 23/05/1972, l'article « Nouvelles brèves » était rédigé comme suit : "Les confédérations syndicales C.G.T.C.F.D.T. et FEN (éducation nationale) ont tenu une réunion commune avec l'intersyndide de l'O.R.T.F. (S.N.R.T. - C.G.T., C.F.D.T., F.S.U., S.N.J.) sur l'initiative de celle-ci pour examiner les menaces qui pèsent sur le service public de l'O.R.T.F. et les ripostes qu'elles entendent organiser en commun. »

En conclusion, l'existence des sigles est due à trois raisons bien définies : le manque de temps, le manque d'espace, la profusion d'organismes. C'est pourquoi il existe le sigle Shape au lieu de Supreme Headquarters of Allied Powers in Europe ; Unesco au lieu de United Nations Educational, Scientific and Cultural Organization; O.N.U. au lieu de Organisation des Nations unies.

\section{LES GEUVRES EXISTANTES}

Plusieurs auteurs ont déjà constaté la nécessité de regrouper tous les sigles et toutes les abréviations en plusieurs listes énumératives et dictionnaires énumératifs traductifs afin de faciliter la tâche de différents groupes quand ils en rencontrent dans des sujets spécialisés :

- Nicolai (Johannis): Tratatus de Siglis Veterum, Lugduni Batavorum, de Swart, $1703,8^{\circ}, 314$ pages.

Cette œuvre, écrite en latin, comprend 49 chapitres qui étudient en détail l'emploi des sigles pour un sujet particulier, illustré par de nombreux exemples : droit, médecine, arithmétique, grammaire, musique, numismatique, sigles sur les ponts, les portes, les monuments funéraires...

- Abréviations de sociétés, conventionnelles et usuelles, publié par le Lloyd Anversois, Anvers, $1926,16^{\circ}, 64$ pages.

Abréviations maritimes, boursières, commerciales (françaises, anglaises, allemandes et espagnoles), abréviations de banques, de corporations, abréviations conventionnelles dans les services d'enregistrement et les services du ministère de l'Intérieur et de la Santé...

- Baudry, Hubert: D.A., Dictionnaire d'abréviations françaises et étrangères, techniques et usuelles, anciennes et nouvelles, Editions de Montligeon, La ChapelleMontligeon (Orne), $1951,8^{\circ}, 157$ pages.

Ce dictionnaire réunit 8000 abréviations dans les domaines suivants : armée, arts, automobiles, aviation banque, cartographie, chimie, commerce, chemin 
de fer, droit, électricité, finances, impôts, industrie, jurisprudence, marine, mathématique, mécanique, médecine, musique, physique, politique, poste, religion, sciences, sports, tourisme, transports, voyages, etc.

- Tramonti, Nino : Dizionario delle sigle e delle abbreviazioni, Busto Arsizio (Unione Bustese degli Industriale - UBI, via Mameli I), 1958, 330 pages.

L'auteur divise son dictionnaire en quatre parties : sigles généraux (organismes politiques, juridiques, économiques, financiers, syndicaux...), monde culturel (terminologie épigraphique, grammaticale, politique, artistique, héraldique...), monde économique (terminologie commerciale, industrielle, agraire, bancaire, maritime...) et appendice (unités d'espace et de temps, symboles chimiques, sigles relatifs à l'automobile...)

- Dictionnaire des sigles et abréviations dans les domaines de la construction, de la sociologie, de l'urbanisme, Centre scientifique et technique du bâtiment, 4, avenue du Recteur Poincarré, Paris - XVI ${ }^{\circ}$, décembre 1968 ( $2^{\circ}$ édition), 123 pages.

Ce dictionnaire comprend plus de 2000 sigles rencontrés dans les domaines les plus variés : établissements publics, services administratifs, banques et autres organismes de crédit, bureau d'études, d'associations d'architectes et, en annexe, les sigles des éditeurs spécialisés.

- Crowley \& Thomas, Acronyms and Initialisms Dictionary, 1965.

C'est en 1950 que fut publié pour la première fois un dictionnaire d'acronymes. On croit que c'est le premier livre rédigé sur le sujet. Très vite, il eut sa place dans le Guide to Reference Books de Minchell (Fourth Supplement), et le Library Journal en indique le titre dans sa liste annuelle de Best Reference Books (Crowley \& Thomas, Préface à la $2^{\circ}$ édition, 1965).

Les auteurs qui ont groupé les sigles et abréviations dans les listes, glossaires ou dictionnaires énumératifs justifient avec raison l'élaboration d'une telle entreprise :

- Abréviations de sociétés, conventionnelles et usuelles, publié par le Lloyd Anversois, 1926 :

«Cette publication permettra de comprendre de nombreuses abréviations ou initiales qui apparaissent dans les journaux et correspondances commerciales. Très souvent, nous nous trouvons face à face avec un véritable casse-tête chinois, tant les initiales se suivent et constituent des obstacles qu'il faut franchir... C'est un grand pas vers une plus grande clarté dans cette montagne de majuscules; comme dans la fable, le lecteur «voit quelque chose, mais ne distinguait pas... »

- Rosenberg, Alexander: Russian abbreviations. A Selective List, Washington, Library of Congress, Reference Department, Slavic and Central European Division, Washington, 1961, 164 pages : 
"La signification de passage entier est obscure parce qu'on ne peut pas déchiffrer une abréviation. Cette liste aidera le lecteur à s'assurer de la signification de nombreuses abréviations... »

- Buttress : World List of Abbreviations :

«We live in an era of abbreviations, or acronyms, a literary disease for which the only cure is extirpation. Such a course is not likely and therefore the only relief is diagnosis without cure. Such a diagnosis has been attempted there.»

L'apparition de groupes économiques et politiques chaque jour plus nombreux, chacun avec ses sigles, rend plus difficile la compréhension des textes. Aussi, avons-nous pensé que la constitution de ce glossaire (voir référence en tête d'article) serait une première étape vers une meilleure compréhension des institutions de ce vaste monde de langue espagnole en continuelle transformation.

\section{CONFUSION ENTRE «ABRÉVIATION 》, «ABRÈGEMENT 》,}

«SIGLE " "ACRONYME » ET «SYMBOLE "

Dans les différents glossaires, dictionnaires, listes..., la confusion entre «Abréviation » et « Sigle » est complète, et très souvent on intitule les œuvres Dictionnaire de sigles et d'abréviations en...

Je crois qu'il est nécessaire de faire ici une distinction entre les deux termes : 1 '«abréviation» est la suppression de lettres dans un mot, de mots dans une phrase afin d'écrire plus rapidement et d'occuper moins d'espace. C'est la réduction graphique d'un mot ou d'un groupe de mots. Par exemple : « km » pour kilomètre, «f.o.b.c.n.p. » pour free on board cars, named point, «f.o.b.c.n.l.f.» pour free on board cars, named point, lighterage free.

Le «sigle» est une initiale ou suite d'initiales qui servent d'abréviation. Par exemple : « C.G.T. » pour Confédération générale du travail.

Certes, la distinction est parfois très difficile à faire. Ainsi, dans la correspondance maritime nous rencontrons :

$\begin{array}{ll}\text { F.A.S. } & \text { Free along sure } \\ \text { G.A. } & \text { General Average } \\ \text { G.B. } & \text { Great Britain } \\ \text { H.H. } & \text { Havre to Hamburg inclusive } \\ \text { N.C. } & \text { New Charter. }\end{array}$

Certains auteurs parlent même d' «acronyme» (ce mot ne se rencontre pas dans le dictionnaire de Paul Robert : Dictionnaire alphabétique et analogique de la langue française). Selon une définition de Alpha Encyclopédique, l'acronyme est un mot formé de sigles correspondant aux premières lettres d'une expression composée et qu'on utilise au lieu de l'expression même pour pallier la prolifération d'objets et d'organismes nouveaux. L'œuvre fait la distinction entre "acronymes alphabétiques » (U.R.S.S. : u, èr, ès, ès), « acronymes phonétiques » (U.R.S.S. : prononcé urs), «sigles acronymiques», à peine déchiffrables par le profane. 
(ADN : acide désoxyribonucléique) ou inintelligibles pour la masse des lecteurs (la nouvelle drogue LSD), « acronymes syllabiques » (Sofrelec: Société française d'électricité), «abréviations acronymiques » dont la composition forme un nouveau mot (Basic English est à la fois « Anglais de base » et, au début, British American Scientific International Commercial English). Alpha Encyclopédique poursuit en disant que TEAM ( équipe » en anglais) est un composé acronymique de Top European Advertising Media, et désigne un groupe d'importants journaux européens liés par un accord ayant trait à la publicité. De cette façon, selon lui, il s'agit de justifier des formations lexicales très souvent ambiguës et arbitraires, afin de conserver la commodité des abréviations sans perturber pour autant la communication.

Les acronymes composés des mêmes lettres se présentent sous les aspects les plus variés. Aussi, un ordre arbitraire a-t-il été établi (Cromley \& Thomas, Acronyms and Initialisms Dictionary, 1970, Editorial Policies, p. XV) :

Straight forms of the acronym

Hyphenated forms of the acronym

Forms of the acronym using the diagonal

Forms of the acronym with ampersand or other symbols
BS

B-S

$\mathrm{B} / \mathrm{S}$

\section{$B \& S$}

Quant à l'«abrègement », ce mot n'a pas encore été admis par l'Académie française. Quoiqu'il soit dans la langue depuis très longtemps, il désigne le procédé par lequel on obtient un nouveau mot en raccourcissant un mot existant : «bus » a été tiré d'«omnibus», «chromo » de «chromolithographie ». Et le nouveau mot a été vite accepté, si vite qu'il a perdu son genre initial ( chromo»). Il y a aussi des « raccourcissements » portant sur des expressions : une «permanente » pour une « ondulation permanente » (on parle alors d'《ellipse »).

Enfin, le «symbole» est un signe arbitraire : les chiffres ou le signe \& pour «paragraphe ».

Voici un tableau comparatif des différentes appellations du phénomène dans différentes langues :

$\begin{array}{llll}\text { Espagnol } & \text { Français } & \text { Anglais } & \text { Américain } \\ \text { abreviación } & \text { abréviations } & \text { abbreviations } & \text { abbreviations } \\ \text { acronimo } & \text { acronymes } & \text { acronyms } & \text { acronyms } \\ \text { sigla } & \text { sigle } & & \text { initialisms }\end{array}$

Allemand

Abkürzungen

Bezeichnungen (kg...)

Symbolen

Akronym

Initial-, Kurz- und Mosaikwörter

Stammelwörter 
Ainsi donc, l'importance de l'usage des sigles croît chaque jour. Quel est l'organisme ou la société, et même le petit groupe sportif provincial, qui n'a pas ses sigles ? Cette profusion de sigles a d'ailleurs éveillé l'intérêt de plusieurs personnes qui ont constitué par conséquent de longues listes énumératives, parfois exhaustives, de sigles dans les domaines les plus variés de la vie politique ou économique. Nous avons fait, ou essayé de faire, une distinction entre les différents vocables qui créent encore de nos jours une confusion parmi les auteurs. Après avoir posé le problème, il convient à présent de définir le mot «sigle». Nous procéderons ensuite à une étude étymologique et historique, qui nous montrera que les sigles étaient déjà connus chez les Hébreux.

\section{CLASSIFICATION DES SIGLES 2}

Les sigles se classent par ordre alphabétique. Cependant, s'ils sont formés des mêmes lettres ou mêmes combinaisons de lettres, on observe généralement les règles suivantes :

1) une lettre capitale précède une minuscule « $A$ » précède « $a$ » et «A.B. » précède «a.B. » et «a.b. »

2) les sigles non ponctués précèdent les sigles ponctués « $A$ » précède «A. »

3) les sigles non séparés par un point précèdent ceux qui le sont par un ou plusieurs points

4) le point précède toute autre forme de ponctuation «A.B. » précède «A.-B. » et «A/B »

\section{DÉFINITION DU MOT «SIGLE»}

Le «sigle» est une lettre initiale (ou suite d'initiales) employée comme signe abréviatif. Paul Robert cite à ce sujet Duhamel (l'Archange de l'aventure, I, page 17) à propos du sigle H.L.M. (voir « habitation », Cit. 10) : « ... ces casernes grisonnantes, que l'on nomme des H.L.M., sigle vulnérable qui signifiait encore " habitations à loyer modéré », mais que l'on pourrait aussi traduire autrement : hommes libres et malheureux. »

Larousse préfère la définition de "groupe de lettres initiales qui constituent l'abréviation de termes employés très souvent ». Exemple : O.N.U., U.N.E.S.C.O., Benelux, ou même « représentation d'un mot au moyen d'une ou plusieurs lettres; mot abrégé. »

Dries Janssen (Van aab to zoo, letterwoorden-book, Uitgeverij Heideland, Vlaamse poekets, $\mathfrak{n}^{\circ} 137$, Hasselt, 1964) définit le sigle comme les initiales d'une série de mots, de façon à former une nouvelle entité, artificielle, mais qui a une résonnance « commode».

2. Crowley, (Ellen T.) \& Thomas (Robert C.) : "Acronyms and Initials Dictionary». 1970 (3e Edition), p. 10. 
Pour Paul-Émile Littré, il s'agit des lettres initiales employées comme signes abréviatifs sur les monuments, les médailles et les manuscrits anciens. Il existe des sigles où on double une même lettre. Le système de tachigraphie ou de sténographie antique consiste en abréviations ou en signes très spéciaux : dans la première catégorie, on consacrait le $\mathbf{C}$ pour signifier «Caius »; le $\mathbf{P}$ pour « Publius »; $\mathbf{D}$ pour « délicat»; S.P.Q.R. pour «senatus populusque romanus», etc. C'est ce que les Romains appelaient litterae singulae, desquels on a formé par abréviation siglae et notre «sigle ». En français, ce mot est masculin : «le» sigle. Littré dit que certains auteurs l'ont fait féminin ( la $»$ sigle), mais il vaut mieux cependant préférer le masculin à cause de son origine neutre.

Pour Maria Moliner, le sigle est la lettre initiale employée comme abréviation de chaque mot et qui constitue un nom. En seconde acception, elle affirme que c'est n'importe quelle abréviation ou écriture où l'on supprime des signes ou des mots pour abréger l'écriture.

Larousse fait encore une distinction entre «sigle simple», qui représente un mot par l'unique lettre initiale de ce mot, et "sigle composé », qui ajoute à la lettre initiale une ou plusieurs lettres du mot.

Hubert Baudry (D.A.: Dictionnaire d'abréviations françaises et étrangères, techniques et usuelles, anciennes et nouvelles », 8000 abréviations, La ChapelleMontligeon (Orne), Editions de Montligeon, 1951) est plus catégorique : «Le sigle est le nom donné à la lettres initiale d'un mot, les autres lettres de ce mot étant supprimées. Les autres lettres qui suivent cette première lettre (ou sigle) sont tout à fait éliminées. »Il donne deux exemples : «J.O.», au lieu de «Journal officiel »; «S.V.P. », au lieu de « s'il vous plaît ».

En conclusion, on peut formuler une définition générale : le sigle est la lettre initiale ou groupe de lettres dont on se sert pour exprimer un mot ou groupe de mots.

\section{ETYMOLOGIE}

Le mot «sigle » vient du bas latin sigla qui signifie «abréviations». Ce mot est une contraction du latin sigilla, pluriel de sigillum, «caractères isolés 》 (A. Beaujean, Dictionnaire de la langue françaie, 1959), d'où, par corruption, singla et «sigle»(P. Larousse, Grand Dictionnaire universel du XIX $X^{e}$ siècle).

P. Robert (Dictionnaire alphabétique et analogique de la langue française) et Albert Dauzat, Jean Dubois et Henri Mitterand (Nouveau Dictionnaire étymologique) pensent que c'est un emprunt (vers 1765) du latin juridique sigla, pluriel neutre, qui signifie «signes abréviatifs ». Ces derniers auteurs citent une formation récente en français : « siglaison ».

Émile Littré (Dictionnaire de la langue française, 1963) et Albert Dauzat (Dictionnaire étymologique) citent l'étymologie du dictionnaire de Trévoux (1771) : le mot provient du bas latin sigla, «signes abréviatifs». 
Maria Moliner (Diccionario de uso del español, 1967) donne l'origine du latin tardif sigla, siglorum, mot toujours au pluriel, et P. Larousse (Grand Larousse encyclopédique en dix volumes, 1964) : du latin siglum, abréviation.

Dans l'abréviation latine, la partie écrite était suivie d'un point (.) : $\mathrm{ABSOLVO}=\mathrm{A}$. ou AB. Parfois, pour éviter l'emploi de ce point, la dernière lettre écrite était tracée un peu plus haut que la précédente : $\mathrm{A}^{\mathrm{B}}$

Le latin écrit, de même que le grec, possédait de nombreuses « suspensions ». Ces abréviations portaient le nom de siglum (?) dérivé de singula littera pro toto (une lettre pour le tout) (V. Vanhaaren, Ce qu'il faut savoir de la sténographie avant d'en entreprendre l'étude, 1947, p. 12). Employées couramment, elles furent officiellement reconnues comme abréviations des prénoms, titres, devises, etc. et admises dans la rédaction des avis publics :

$$
\begin{array}{ll}
\text { T. } & =\text { Titus } \\
\text { M. } & =\text { Marcus } \\
\text { P.C. } & =\text { Patres conscripti } \\
\text { S.P.Q.R. } & =\text { Senatus populusque romanus }
\end{array}
$$

Il convient cependant de noter que tous les systèmes sténographiques actuels profitent eux aussi largement de ce mode d'abréviation ; ils emploient souvent un SEUL signe - généralement le premier - pour abréger un sténogramme entier représentant un mot très fréquent :

$$
\begin{aligned}
& \mathrm{N}=\text { nous } \\
& \mathrm{V}=\text { vous } \\
& \mathrm{B}=\text { bien }
\end{aligned}
$$

Ce signe isolé s'appelle en terminologie sténographique «sigle » (siglum) confondu trop souvent avec «sténogramme arbitraire » qui représente tout autre chose.

\section{UN PEU D'HISTOIRE}

Selon certains, les Hébreux connaissaient les «sigles». Les Grecs les ont utilisés - Xénophon fut le premier à les employer - et les ont transmis aux Romains. Ennius, Tullius Tiron, Aulus Persius Flacus, Lucius Annaeus, Sénèque et Tibère connaissaient une centaine de symboles et de sigles. Cicéron les appelait singulae litterae. Par phénomène d'abréviation, on a obtenu siglae et en espagnol siglas. Ce fut Tiron, l'affranchi de Cicéron, qui, selon la tradition, a systématisé l'emploi de cette forme de notation abrégée.

Les chrétiens ont écrit sur les murs des catacombes le mot Ichtus, mot grec qui signifie «poisson ». C'était un mot code formé par les lettres initiales des mots

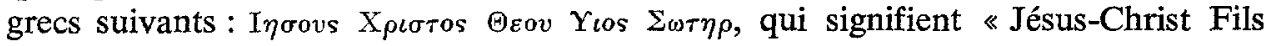
du Dieu Sauveur ».

Ponce Pilate fit écrire sur la croix de Jésus-Christ Inri, pour Iesus Nazarenus Rex Iudaeorum (Jésus de Nazareth, roi des Juifs). Et certaines personnes prétendent qu'au début du christianisme les abréviations religieuses étaient dictées par un sentiment de respect envers Jésus-Christ dont on ne pouvait ni écrire le nom ni le prononcer: IHS (les trois premières lettres grecques du nom de Jésus). 
Les manuscrits, les inscriptions funéraires, les médailles présentent de nombreux «sigles ». Voici, par ordre alphabétique, quelques sigles très employés :

A.

A.A.A.

A.M.

A.V.C.

C.VIR.

C.C.V.V.

D.

D.M.

D.M.S.

D.O.M.

D.S.P.

G.D.N.

I.H.S.

I.O.M.

L.

N.P.

P.M.

Q.D.E.R.F.P.

S.

S.C.

S.P.Q.R.

S.T.T.L.

V.F.

V.S.L.
Absolvo

Auro, argento, aere

Amicus

Anno Urbis Conditae

Centumvir

Clarissimi viri

Dies, o divus, o decreto

Dis manibus

Dis Manibus sacrum

Deo Optimo maximus ( = a Dios muy bueno, my grande : à Dieu très bon, très grand )

De sua pecunia

Genio domini nostri

Jesus Hominum Salvator

Jovi Optimo Maximo

Libertus. o Liberta

Nobilissimus puer

Pontifex maximus

Quod de ea re fieri placuit

Sacrum

Senatus Consultum

Senatus Populusque Romanus

Sit tibi terra levis

Vivus fecit

Votum solvit libens

Les Anciens employaient aussi les «sigles »dans la transcription des lois et des décrets. Ces abréviations des Romains appliquées à des termes courants étaient faciles à retenir et à traduire, mais cette facilité de lecture devenait toute relative, dès qu'une seule lettre pouvait avoir plusieurs significations :

A.

B.

C. absolvo, ager, amicus, animus, anno, ante...

bis, bona, bonus, Brutus...

caput, castra, Caesar, candidatus...

La première lettre n'étant pas toujours suffisante, on lui adjoignait parfois une seconde :

SA.

Salus, sacerdos...

QS.

quasi

Certaines lettres isolées étaient barrées par un trait (ou par une lettre) pour leur donner « arbitrairement » un sens particulier et fixe :

$$
\begin{array}{llll}
\mathrm{I} & \text { (barré) } & = & \text { inter } \\
\mathrm{D} & \text { (barré) } & = & \text { dixit } \\
\mathrm{L} & \text { (barré) } & = & \text { libra }
\end{array}
$$

Aujourd'hui, le signe $£$ est resté pour désigner la Livre Sterling anglaise. 
Il existait un troisième moyen abréviatif : la « contraction $»$. On écrivait le début du mot (parfois le radical seul) ainsi que la fin du mot, le milieu étant délaissé : considerat $=C^{\text {at }}$. Aujourd'hui, les Grecs décrivent : XPS $=$ Chistus ; les Anglo-Saxons : Xmas, pour Christmas (Noël). Nous connaissons aussi des abréviations contractes : $\mathrm{C}^{\mathrm{ie}}=$ compagnie.

Dans l'Antiquitế, on fit un usage de plus en plus abusif des abréviations. Elles apparurent si nombreuses dans la rédaction des actes officiels que le contexte de ceux-ci put parfois être interprété de différentes manières. La difficulté de déchiffrement des textes devint d'autant plus grande qu'au lieu de suivre un système uniforme d'abréviations, chaque écrivain s'en créait un particulier pour son propre usage. Aussi l'empereur Théodose II (408-450) et après lui Justinien I (527-565) interdirent leur emploi dans ces actes, afin d'éviter les abus.

Dans l'Antiquité la coutume de copier les livres à la main développa l'emploi des abréviations. Les lettres abrégées étaient surmontées d'un petit trait ( lus »), qui existe encore aujourd'hui dans le «tilde» de la $\tilde{\mathrm{n}}$ espagnole, l'accent circonflexe des voyelles longues françaises et les points diacritiques que l'on met sur les consonnes d'autres langues.

Les abréviations continuèrent donc à évoluer et leur nombre à augmenter. Ennius (poète latin né en 239 av. J.C.) en avait complété la liste, tout en leur attribuant déjà des signes spéciaux.

Le grand orateur romain, Cicéron, avait un secrétaire et ami, Tiron, à qui il apprit à se servir habilement des signes abréviatifs d'Ennius qu'il employait déjà lui-même. Tiron les perfectionna et inventa, croit-on, 200 signes nouveaux pour présenter les prépositions. Ces abréviations servirent de base au système tachygraphique connu sous le nom de «Notes tironiennes».

Agrippa Vipsanius apporta, pour sa part, 400 nouvelles abréviations pour les radicaux, les préfixes et les suffixes.

Aquila créa 1270 abréviations. Vers l'an 45 après J. C. (40 ans après la mort de Tiron) 3000 notes furent ajoutées aux précédentes et Sénèque (le philosophe) en trouva encore 300 autres. Ce fut d'ailleurs lui qui rassembla dans un corpus toutes les notes connues jusqu'à cette époque, soit environ 5000 .

Les notes tironiennes furent complétées vers 175 après J. C. Elles nous ont été transmises dans un ouvrage appelé Commentarii notarium tironarium (CNT) qui comprenait 6 " commentaires » numérotés de 1 à 6 , chacun composé de 132 tablettes ou listes de notes. Les 83 premières tablettes (C.N.T. 1 et 2) semblent contenir les 5000 abréviations rassemblées par Sénèque; les suivantes ont été établies dans la Gaule.

Au IX ${ }^{e}$ siècle, les trois fils de Louis le Débonnaire signèrent le traité de Verdun, réglant le partage de l'empire carolingien et enlevèrent ainsi à celui-ci sa vie propre. Les mouvements de population qui en résultèrent furent une des conséquences de la décomposition du latin, ce qui provoqua sa disparition en 
tant que langue populaire. Le peuple, ne comprenant plus cette langue, l'étude des notes, crées pour elle, devint inutile. Elles ne furent plus employées, à la fin de la Renaissance carolingienne, que dans les couvents. Les documents de cette époque rédigés en Notes, sont en général des copies de psaumes, des règlements intérieurs de couvents, des ouvrages de théologie, des recettes médicales, etc. On ne se servait plus des notes pour la prise des discours, mais comme écriture de «copie» pour épargner le parchemin dont le coût était très élevé. Les Notes tombèrent dans l'oubli. Le dernier document en N.T. semble dater de 1067.

Ce fut entre le $\mathrm{IX}^{\mathrm{e}}$ et $\mathrm{le} \mathrm{XV}^{\mathrm{e}}$ siècle, dans les écritures caroline et gothique, que l'on rencontre encore plus d'abréviations. Les systèmes abréviatifs étaient le sigle, la contraction, la suspension ou le tronquement (mot inachevé), les lettres sous-écrites, des signes spéciaux.

De nombreuses erreurs historiques sont dues à des «sigles » mal écrits ou mal lus. Les copistes, à certaines époques, ont exagéré très fort l'emploi des «sigles». Ainsi le manuscrit de Virgile, connu sous le nom de «Virgile de Asper », qui passa de l'abbaye de Saint-Germain-des-Prés à la Bibliothèque nationale, présente d'importants fragments écrits en «sigles», sans que l'on puisse soupçonner le but réel de cette transcription tout à fait incompréhensible si on ne la lit pas avec l'aide du texte complet.

$\mathrm{Au} \mathrm{XI}^{\mathrm{e}}$ siècle, selon le «Nouveau traité de diplomatie », on n'avait pas oublié cette façon d'abréger l'écriture. Le fameux cartulaire d'Angleterre, rédigé sur l'ordre de Guillaume le Conquérant, en est une preuve. Ce manuscrit de deux volumes, que les Anglais appellent «Domesday-Book», fut écrit en lettres anciennes et en «sigles». Ces «sigles», cependant, ne sont pas, et de loin, aussi fréquents que dans le «Virgile de Asper».

On employait aussi les «sigles » pour distinguer les livres, pour indiquer la numérotation des chapitres et des cahiers des manuscrits. De même, on exprimait la valeur des poids avec différentes lettres des alphabets grec et latin. Les médecins, dit Pierre Larousse (Grand Dictionnaire universel du $X X^{e}$ siècle), emploient aujourd'hui encore, dans leurs prescriptions, des «sigles» dont l'usage remonte très loin. Il terminc en disant que, parmi les autres sigles qui existent encore, il faut signaler celui qui accompagne, dans les actes publics, la signature de l'Empereur d'Autriche : «M. P.», «Motuproprio » (de notre propre mouvement, de notre propre volonté). Il convient de citer ici l'ouvrage de Johannis Nicolai, écrit en latin Tratatus de siglis veterum (Lugduni Batavorum, de Swart, 1703).

De nos jours, les abréviations et les signes sont d'une utilisation fréquente, même excessive. La nécessité d'un langage technique toujours plus complexe et les exigences d'un échange de plus en plus rapide d'information de tous genres expliquent ce phénomène. Il s'agit surtout de termes en rapport avec les domaines politiques (partis, groupements, mouvements), scientifiques, militaires, commerciaux et, plus généralement, de la multitude d'organismes. 
VIII. FORMATION, PRONONCIATION ET CAPITALISATION DES SIGLES

La formation des sigles a posé, et pose encore, des problèmes, surtout l'emploi du point de séparation entre les diverses lettres du sigle.

Il n'existe aucune règle absolue pour la formation des sigles. En général, il est suivi d'un point après chaque initiale (Baudry, Dictionnaire d'abréviations françaises et étrangères, techniques et usuelles, anciennes et nouvelles).

C.E.T.U.S. : Cooperativistas en Empresas de Transportes Urbanos Salvadoreños (El Salvador).

C.N.T.C. : Confederación Nacional de Trabajadores Campesinos (Bolivie).

P.R.G.I. : Partido Republicano Guanacasteca Independiente (Costa Rica).

Il peut être suivi sans point, comme si l'ensemble constituait un seul mol :

CODELCO : Corporación del Cobre (Chili).

SABENA : Société Aéronautique Belge d'Exploitation de la Navigation Aérienne (Belgique).

Dans ce cas, on peut l'écrire comme un simple mot, c'est-à-dire avec une majuscule suivie de minuscule :

RENFE ou Renfe: Red Nacional de Ferrocarriles (Espagne).

"Quisiera simplemente acentuar la opinión publica de desagrado sobre el deficiente servicio de la Renfe (Red Nacional de Ferrocarriles) y su total falta de atención hacia los viajeros que confián en ella y que no siempre son tratados como clientes » Revista SP, Madrid, $\mathrm{N}^{\circ} 437$, page 7).

Certains sigles sont tellement entrés dans l'usage qu'ils ont formé de véritables mots, se confondant ainsi avec les mots du dictionnaire :

Laser : Light Amplification by Stimulation Emission of Radiation

Radar : Radio Detection And Ranging

On peut remarquer l'évolution de l'écriture des sigles à travers les sigles d'organisations internationales :

$$
\begin{aligned}
& \text { U.N.E.S.C.O. } \rightarrow \text { UNESCO } \rightarrow \text { Unesco } \\
& \text { O.N.U. } \rightarrow \text { ONU } \rightarrow \text { Onu }
\end{aligned}
$$

Parfois on adopte les premières lettres des mots les plus importants pour former un nouveau mot prononçable :

CONAHOTU : Corporación Nacional de Hoteles y Turismo. (El Salvador) INQUITEX : Industrias Quimicas Textiles. (Espagne)

SEFANITRO : Sociedad Española de Fabricación de Abonos Nitrogenados.

On prononce généralement ce genre de mots en énonçant séparément chaque lettre dans le premier cas et en un seul mot composé de différentes syllabes dans le second; cependant, il n'es pas rare de les énoncer en un mot dans le premier, quand l'association des lettres le permet (O.N.U.) Ainsi, J.O.C. est lu «ji-o-cé» ou «jok», et non «Jeunesse ouvrière chrétienne ». 
Crowley \& Thomas, dans leur Acronyms and Initialisms Dictionary (Editorial Policies), écrivent : «Occasionally, an initialism will acquire an unofficial stray letter or sound which never appears in print, but which renders the term pronounceable :

SNCC : Student National Coordinating Committee (is widely pronounced «Snick»), Dans des cas semblables, ajoutent les auteurs, il convient de prêter une grande attention.

Pour Zumthor (Abréviations composées), les sigles dont les lettres s'épellent successivement selon leur appellation traditionnelle dans l'alphabet (S.D.N. = ès-dé-èn) forment le groupe «type létrique à valeur alphabétique »; le second groupe, dont les lettres s'assemblent en syllabes selon leur ordre de succession (U.N.E.S.C.O. : U-nès-co), forme le «type létrique à valeur syllabique ». Il poursuit : «La frontière qui sépare ces groupes n'est pas nette. Il est évident que les abréviations formées uniquement de consonnes appartiennent au premier groupe. On renonce à prononcer en syllabes les abréviations formées uniquement de voyelles, ou qui comprennent une série de deux ou plusieurs voyelles... Pour les abréviations qui ont une alternance de consonnes et de voyelles, la tendance générale de toutes les langues est d'éviter la prononciation syllabique si elle conduit à la formation de phonèmes absurdes. Mais à ce sujet, il existe de nombreuses exceptions. C'est ainsi que l'anglais prononce alphabétiquement YMCA qui pourrait cependant se prononcer parfaitement en syllabes. Indifféremment on entend en français : ( o-èn-u ») et ( $(0-n u »)$ pour ONU. »

Certains sigles se transforment finalement en de vrais concepts (parfois appelés acronymes) qui peuvent dans certains cas prendre la marque du pluriel, se décliner, selon les règles grammaticales de la langue considérée. Cette dérivation est un signe de lexicalisation avancée. Le sigle peut être substantif ou un adjectif. Dans le premier cas, il est précédé de l'article défini dans tous les cas normalement exigés par la syntaxe des différentes langues :

APRA : Alianza Popular Revolucionaria Americana (Pérou).

$$
\rightarrow \text { aprista }
$$

C.O.P.E.I. : Comité de Organizacion Politica Electoral Independiente (Venezuela)

$\rightarrow$ copeyano

«Si el gobierno copeyano no es capaz de satisfacer las aspiraciones del MIR (Movimiento de la Izquierda Revolucionaria), expresión de la voluntad de amplios sectores democráticos y revolucionarios de Venezuela, determinará que su actual propaganda en torno a la pacificación no es más que una campaña demagógica destinada a engañar al pueblo y a sembrar la ilusión de una paz que no existirá » (Revista SP, Madrid, $n^{\circ} 446$, page 29 ).

"The moderate, pro-AD leadership of the national labor movement has lost ground recently, with both Copeyno and extreme leftist leaders chipping away at the once monolithic AD control $\gg$ (Ben G. Burnett and Kenneth F. Johnson: Political forces in Latin America : Dimensions of the Quest for Stability, 1968). 
MAPU : Movimiento de Acción Popular Unitaria (Chili).

$\rightarrow$ mapuche

M.I.D. : Movimiento de Intergración y Desarrollo (Argentine).

$\rightarrow$ midista

«Con relación a la reorganización y en la certeza de contar con los cuadros midistas bien cohesionados... »(Análisis, $\mathrm{n}^{\circ} 544$, page 16 ).

M.I.R. : Movimiento de la Izquierda Revolucionaria (Venezuela).

$\rightarrow$ mirista

«El P.C.V. (Partido Comunista Venezolano) mantendrá la misma lucha por la legalización de la miristas porque las cosas no han cambiado bajo ningún aspecto en esa materia 》 (Revista SP, $\mathrm{n}^{\circ}$ 460, 20 juin 1969, page 22).

«Both the regular of the PCV and the more radical Miristas contributed to the violent antigovernmental campaign of the extreme left» (Ben G. Burnett and Kenneth F. Johnson : Political Forces in Latin America: Dimensions of the Quest for Stability, p. 225).

Les dirigeants miristas dont la parenté politique avec les leaders du M.I.R. vénézuélien ou du M.O.E.C. colombien était évident ».

(Marcel Niedergang: «Les vingt Amériques latines», Tome 2, page 131).

Parfois le sigle remplace l'adjectif correspondant :

«... el monstruo (yanki) resoplaba impunemente por las calles de Lima desde que los militares peruaons desenvainaron las espadas ante una petrolera que los militares peruaons desenvainaron las espadas ante una petrolera USA... » (Revista SP, $n^{\circ} 460,20$ juin 1969, page 22).

\section{Emploi des points}

L'emploi de points entre chaque lettre est très discuté. Selon une théorie on ferait une distinction entre les sigles qui s'énoncent en prononçant chacune des initiales (c'est-à-dire les sigles qui s'épellent) et les sigles assimilables à un mot conventionnel prononçable. Dans le premier cas, on pourrait employer des points et, dans le second, on pourrait s'en dispenser.

Il semble, cependant, que la tendance actuelle soit à la généralisation de la suppression du point, ce qui satisfait probablement à des désirs de simplification et d'esthétique à la fois. D'autre part, cette tendance est accentuée par le recours de plus en plus fréquent à des procédés acronymiques comme dans MATESA (Maquinaria Textil del Norte de España, Sociedad Anonima).

Il faut noter aussi qu'il n'est pas rare de voir l'emploi simultané des deux cas dans un même texte (E.T.A., ETA) :

«Después de permanecer 72 horas detenido por orden de la Authoridad Judicial Militar que entiende de las actividades y delites de la E.T.A., el 
vicario apostólico de la diocésis de Bilbao, José Angel Ubieta, sobre el que habian recaido ciertas sospechas de colaboración...» «Sabemos, sin embargo, que un nombre fue fríamente asesinado por la espalda y que el presunto asesino, Miguel Echevarría, de 23 años de edad, perteneciendo a la ETA, logró escagar... 》 (Revista SP, $\mathrm{n}^{\circ} 445$, page 29).

« Enasa naturalmente, en cada país donde las circunstancias lo exigen, ha presentado su proposición para seguir en aquellos mercados en la forma que más concuerde con las disposiciones de los respectivos gobiernos... »

«... destacó que, pese a las condiciones particularmente difíciles del año 1968 , el ejercicio de E.N.A.S.A. ha terminado brillantemente... » (Revista $\mathrm{SP}, \mathrm{n}^{\circ} 458$, page 49 ).

Il semblerait donc que le sigle avec points soit de rigueur dans le titre ; celui sans points dans le texte rédigé.

Néanmoins, quand il s'agit d'une organisation de caractère national, il faut répéter le sigle intégralement et il convient, comme c'est la règle pour les textes rédigés directement dans la langue, de le faire suivre de la traduction, la première fois qu'il est mentionné, de sa dénomination en toutes lettres dans la langue d'origine de l'organisation ou organisme qu'il représente. Tout le texte doit rappeler en toutes lettres l'appellation à laquelle se rapporte un sigle quand on le mentionne pour la première fois.

À ce propos, J. de Maguerie disait dans son Abréviations et sigles économiques et sociaux (p. 1):

«Depuis quelques années, le défaut anglo-saxon des abréviations s'est répandu dans notre pays.

Nous pouvons le regretter, mais c'est un fait auquel nous devons nous habituer le mieux possible.

Dans cette optique, nous croyons utile de mettre à la disposition de nos lecteurs un vocabulaire des principaux sigles utilisés en matière d'économie, de finances et de sociologie.

Cependant nous voudrions espérer que ce travail ne soit pas considéré comme un stimulant à employer chaque fois, et à tort et à travers, des abréviations et sigles qui bien souvent sont d'un emploi moins facile que le titre même qu'il veut exprimer.

Finalement nous aimerions recommander aux utilisateurs des sigles qu'ils ne les emploient dans un texte qu'après avoir rédigé, au moins une fois, les titres auxquels ils se réfèrent, en les faisant suivre de leurs initiales entre parenthèses, étant donné que de mêmes abréviations peuvent désigner des organismes différents. 》

De même F. A. Buttress, dans son World List of Abbreviations (Leonard Hill, London, 1966, $3^{\mathrm{e}}$ édition, Introduction : p. iii) :

"The modern tendency to abbreviate, and the failure of many editors to record at least once the full meaning of the abbreviations make matters 
increasingly difficult for the reader. The saving of space by the use of abbreviations is fully appreciated, especially in abstracting journals, but for the benefit of the reader and increased usefulness of the publication, editors should realise the importance of listing the full meaning of such abbreviations either at the beginning or end of the publication or abstracting section. Frequently the abbreviation is given in full when first mentioned and thereafter in the abbreviated form. In a small publication this is satisfactory but in larger publications it may entail looking back at each page; hence the advantage of listing the abbreviations at the beginning or end of the publication. 》

En conclusion, nous voyons qu'il n'existe aucun critère uniforme quant à l'usage du point de séparation entre les diverses lettres du sigle. En règle générale, on suit l'usage consacré par la pratique : l'écriture sans point, surtout quand il s'agit d'institutions ou d'organismes qui par leur ancienneté ou leur importance sont très connus. Dans ce cas, tout le monde accepte le sigle comme un concept en soi (voir ALALC, APRA, COMIBOL, qui sont plus connus que sous leur complète dénomination).

Étant donné la tendance moderne de tout faire rapidement, l'emploi du sigle se généralise jusqu'à être accepté sans point dans des cercles de plus en plus grands, c'est-à-dire comme un mot indépendant.

\section{CAPITALISER OU NE PAS CAPITALISER ?}

Il est relativement rare, et même moins courant, d'écrire les sigles sous une autre forme que les lettres capitales, sans points. Il existe parfois des exceptions, surtout dans la littérature scientifique et technique (noms d'éléments chimiques, par exemple), mais il semble que la tendance générale soit à l'usage des capitales et à l'omission de la ponctuation. La capitalisation des sigles pour les noms d'usage courant, tel que « TV » pour « télévision », est un cas typique.

Les éditeurs ont étudié les raisons de cette tendance à l'emploi des capitales non suivies de point, mais sans succès. Il est significatif, cependant, de faire remarquer que l'équipement employé pour l'enregistrement des données et/ou les communications - tel que les télétypes, les calculatrices électroniques, les cartes perforées, etc. - utilise uniquement (presque toujours) des lettres capitales et, sauf pour le télétype, cet équipement est peu habilité à utiliser caractères, ponctuation et espace.

\section{PARTICULARITÉS DE LA FORMATION DES SIGLES}

1) Répétition de lettres

On représente généralement un nom pluriel par une répétition des initiales : «EE.UU.» (Estados Unidos), mais il n'est pas rare de voir le mot au singulier :

«E.U.», ou les sigles anglais «USA $»$ : 
«Claro que puede existir fundados motivos para dudar de la buena fe de algunos para cumplir sus compromisos, sin la que éstos serian completamente inutiles, pero si esto es así y la desconfianza no apunta a los E.U., como es posible que en este país tanta influencia puedan tener ésos que se llaman pacifistas y aun se acepte sin escándalo este calificativo, que en buena lógica no les corresponde? $\gg$ (Revista $\mathrm{SP}, \mathrm{n}^{\circ} 455$, page 5).

\section{2) Emploi de mots-outils (palabras de conexiones)}

La structure des sigles est en principe très simple : une lettre pour chaque mot abrégé. Mais il existe entre les différentes langues des différences d'emploi dues à leur système morphologique et syntaxique. Les langues flexionnelles et celles où la composition se fait sans l'aide de prépositions peuvent abréger plus facilement tous les éléments de leurs expressions composées. En revanche, les langues romanes, où prolifèrent les mots-outils, doivent très souvent les exclure de leurs abréviations. La tendance actuelle à toutes les langues est de limiter le nombre de mots vides, et même de les éliminer complètement :

A.N.D.I. : Associación Nacional de Industriales (Colombia).

C.I.C.Y.P. : Consejo Interamericano de Comercio y Producción.

FIDEL : Frente Izquierda de Liberación (Uruguay).

\section{3) Terminaison $\ll-S . A . »$}

Beaucoup de sigles, représentant des cercles commerciaux, se terminant par deux lettres qui forment une syllabe «S.A. » ou «SA》 (Sociedad Anonima) :

INACSA : Industria del Acetato de Celulosa, Sociedad Anónima. (Espagne)

L.C.S.A. : La Calzadora, Sociedad Anónima. (El Salvador)

Parfois, on met une virgule entre la première partie du sigle et «S.A.» :

S.T.E.C.K., S.A. : Sindicato de Trabajadores de la Empresa Kimberly Clark, Sociedad Anónima (El Salvador).

\section{4) Élément final mobile}

Certains sigles ont un élément final mobile, qui s'exprime uniquement si le contexte ou l'intention l'exige. «P.C. » (Partido Comunista, Parti Communiste) est un de ces sigles universels. Il suffit d'ajouter une autre lettre pour désigner le pays :

P.C.B. Partido Comunista Boliviano

P.C.C. Partido Comunista de Cuba

P.C.D. Partido Comunista Dominicano

P.C.E. Partido Comunista de Espana

P.C.V. Partido Comunista Venezolano

5) Différentes interprétations pour un même sigle

C.U.T. Central Única de Trabajadores de Chile

CUTCH Confederación Única de Trabajadores de Chile 
F.A.R. Fuerzas Armades Rebeldes

$\begin{array}{ll} & \text { Fuerzas Armadas Revolucionarias } \\ \text { HUNOSA } & \text { Hullera y Energía del Norte, S.A. }\end{array}$

(Guatemala)

(Espagne)

Empresa Nacional Hullera del Norte, S.A.

6) Mêmes sigles pour deux ou plusieurs pays

a) E.L.N.: Ejército de Liberación National (Colombie)

Ejército de Liberación Nacional (Mexique)

F.A.R. : Fuerzas Armadas Revolucionarias (Argentine)

Fuerzas Armadas Revolucionarias (Guatemala)

$\begin{array}{ll} & \text { Fuerzas Armadas Revolucionarias (Pérou) } \\ \text { C.T.C. : } & \text { Confederación de Trabajadores de Colombia }\end{array}$

Confederación de Trabajadores Cubanos

Control de Tráfico Centralizado (Espagne)

b) I.N.I. : Instituto Nacional de Industria (Espagne)

P.R. : $\quad$ Partido Radical (Chili)

Partido Reformista (République Dominicaine)

Partido Revolucionario (Guatemala)

7) Deux sigles pour une même organisation

$\left.\begin{array}{l}\text { C.O.B. } \\ \text { COBOL }\end{array}\right\}$ Central Obrera Boliviana

8) Erreurs

On omet parfois de répéter une même lettre dans un sigle :

F.O.R.P.P.A. \} Fondo Ordenador y Regulador de productos y Precios FORPA $\}$ Agricolas (Espagne)

Les points se justifient quand il s'agit d'une série d'initiales (la première lettre de chaque mot) :

M.I.N.C.I.N. : au lieu de MINCIN (Ministerio del Comercio Interior-Cuba)

M.I.N.F.A.R. : au lieu de MINFAR (Ministerio de las Fuerzas Armadas Cuba

Amérique latine, $\mathrm{n}^{\circ} 13$, juin 1968 , page 20 )

Dans le cas suivant, il n'est pas nécessaire de mettre des points :

CO. FE. TR.O.V.: Comité para la Federación de los Trabajadores Organizados de Venezuela

CON. CA. NA. CO. : Confederacion de Cámaras Nacionales de Comercio (Mexique) 
9. Changement de nom

Un Conseil de Ministres peut changer la dénomination d'un organisme :

HENOSA $\rightarrow$ HUNOSA : Empresa Nacional Hullera del Norte, Sociedad Anónima (Espagne)

La même chose peut arriver à la suite d'un décret d'unification :

F.E.T. y de las J.O.N.S. : Falange Española Tradicionalista y de las Huntas de Ofensiva Nacional-Sindicalista

10. Mots charnières

C'est un phénomène typiquement belge :

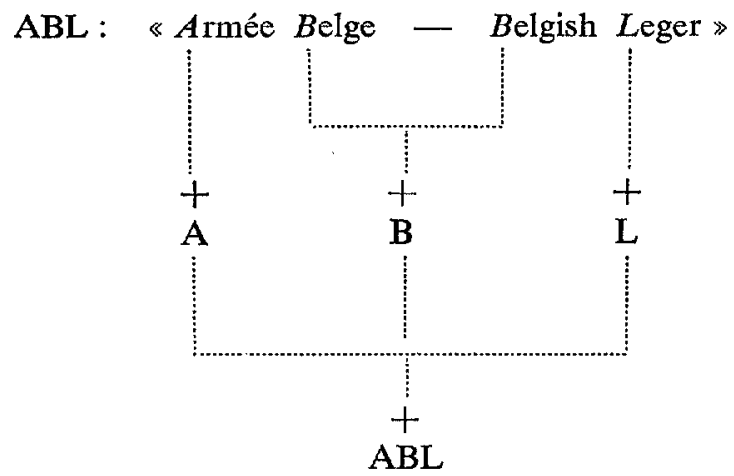

GNG : «Gendarmerie Nationale Gendarmerie ».

\section{1) Sigles prédestinés}

Une des raisons d'être d'un sigle peut être par exemple celle de donner aux initiés un moyen mnémotechnique. Ainsi, le sigle MIRACLE ( $\mathrm{m}$ i r k $1 »$ ) d'un ordinateur inventé par les Hollandais (au lieu de «Mokums Industrial Research Automatic Calculator for Laboratory and Engineering 》).

Il apparaît également qu'un sigle peut avoir un but plus ou moins publicitaire (MIRACLE).

Il existe des sigles-symboles, destinés à avoir un impact sur le public :

\section{ZON : Ziekenomroep Nederland}

Ce mot néerlandais signifie «soleil » et «ziekenomroep » est une émission de radio pour les malades. Ce sigle doit donc représenter la joie et la lumière dans les chambres des malades.

L'auteur hollandais Dries Janssen donne d'autres exemples:

WOMAN : sigle anglais qui signifie «femme», pour « $W$ orld Organization of the Mothers of $A$ ll Nations »

(Organisation mondiale des Femmes de tous les pays). C'est un symbole évident. 
Il est d'autres exemples :

FIDEL : Frente Izquierda de liberación (Uruguay). Analogie avec le nom de Fidel Castro.

F.U.E.G.O. : Frente Unido de Estudiantes Guatemaltecos. Analogie avec le pouvoir du feu.

M.A.N.O. : Movimicnto Anticomunista Nacional Organizado (Guatemala). Analogie avec la Main noire, la Mafia, etc.

SMOG : ce mot signifie «brouillard » en anglais. En Russie : «L'association de génies la plus jeune ». À partir de 1960 apparut parmi la jeunesse universitaire et intellectuelle un mouvement littéraire-politique dont l'unique moyen d'expression possible se limitait à une série de revues littéraires publiées en éditions de 100 à 300 exemplaires. C'est ainsi que surgirent une pléiade de publications plus ou moins clandestines. Le groupe littéraire qui se fit remarquer dans les années 1964-1965 fut connu — par les seuls intiés qui fréquentaient les cercles artistiques et littéraires — par les sigles SMOG, groupement qui joua un rôle important non seulement par ses actions répétées de protestation politique comme dans le cas de la manifestation organisée en faveur de la liberté des écrivains Sinjavskij et Daniel, mais aussi et surtout parce qu'il représenta le premier essai de constitution, bien que ce fut au niveau artisanal, d'une première maison d'édition indépendante de l'État.

L'objet mnémotechnique ou symbolique des sigles a donc un but pratique. $\mathrm{Ce}$ but peut aussi être euphonique (dans la publicité) :

SARMA : Société Anonyme de Récupération des Matériaux Abimés.

\section{2) Les termes «préfabriqués » gagnent en popularité}

Aujourd'hui, il semble bien que l'on considère l'acronyme comme un facteur clé dans le succès de projets, mouvements, et autres genres d'activités dont l'efficacité dépend de l'attrait qu'il exerce sur les yeux ou l'imagination du public. Ils sont formés de façon à transmettre une certaine impression pour un certain but.

Des exemples pris dans le domaine de l'écologie et de la conservation de la nature démontreront l'étendue d'emploi de ces acronymes préfabriqués :

GASP : Greater ( + nom de ville) Alliance to Stop Pollution.

SCARE : Students Concerned About a Ravaged Environment.

ENACT : Environmental Action.

Des activités pour et contre l'éducation sexuelle (voir Crowley \& Thomas : Acronyms and Initials Dictionary, 1970, Préface à la $3^{e}$ édition, page vii) ont donné :

NOMS : Mothers for Moral Stability.

PAUSE : People Against Unconstitutional Sex Education.

POSSE : Parents Opposed to Sex and Sensitivity Training.

POSE : Parents Opposed to Sex Education.

SOS : $\quad$ Sanity on Sex. 


\section{3) Un peu d'humour}

Il est une autre tendance qui s'accentue peut-être: on introduit un peu d'humour, heureusement, dans la composition de certains termes! Dans le combat épique entre les jupes «midi » et «mini », par exemple, on trouve des antagonistes de ce genre :

GAMS : Girls Against More Skirt.

SNACK : Society of Males Who Appreciate Cute Knees.

POOFF : Preservation Of Our Feminity and Finances (filles opposées à l'achat de longues jupes qui remplaceraient une garde-robe pleine de minis).

OGLE : Organization for Getting Legs Exposed.

PEEK : People for the Enjoyment of Eyeballing Knees.

\section{PROBLÈMES DE TRADUCTION}

La traduction des sigles pose un problème au traducteur-interprète et au journaliste. Traduit-on le sigle? Si oui, il faut rechercher son équivalent dans la langue d'arrivée; si non, faut-il le citer dans sa langue de départ? Qu'en est-il alors de sa compréhension? Peut-on donner une traduction plus ou moins équivalente entre parenthèses? L'interprète aura-t-il le temps nécessaire de le transposer?

On peut faire suivre le sigle de sa version dans la langue nationale. Cette traduction ne peut être qu'approximative étant donné que la plupart de ces dénominations comprennent des termes plus ou moins en rapport avec le vocabulaire institutionnel du pays étudié. Des sigles comme NASA (National Aeronautic and Space Agency) ou CIA (Central Intelligence Agency) en anglais, GESTAPO (Geheime Stadts Polizei) en allemand, etc., n'ont pas de correspondants rigoureux en français.

La traduction, non seulement de la dénomination de l'organisation, mais aussi du sigle correspondant, s'impose au contraire dans les pays bilingues, par exemple en Belgique. Si, dans un texte français, on rencontre le sigle T.V.A. (Taxe à la valeur ajoutée), il convient de le traduire en flamand par B.T.W., et réciproquement, puisqu'il s'agit ici d'expressions officielles reconnues (en espagnol, on le traduit par I.V.A. : Impuesto sobre el Valor Ahadido).

Autre exemple: S.N.C.B. (Société nationale des chemins de fer belges) se traduit en flamand par N.M.B.S. (Nationale Maatschappij der Belgische Spoorwegen).

Il semble que la traduction des sigles d'organisations internationales soit obligatoire. Cependant, il n'en résulte pas nécessairement que le sigle correspondant change selon la langue et certains sigles s'emploient, quelle que soit la langue, sous la même forme, née de la dénomination dans une langue déterminée de l'organisation étudiée :

- le sigle C.E.E. (Communauté économique européenne) a son correspondant en anglais : «E.E.C.» (European Economic Community) ; 
- le sigle O.T.A.N. (Organisation du traité de l'Atlantique-Nord) correspond à l'anglais NATO (North Atlantic Treaty Organization). En Espagnol, les deux sigles sont employés, bien que OTAN soit le plus courant.

«Desde el punto de vista dialéctico el Pacto de Varsovia se estableció porque los paises del Pacto del Atlántico Norte - OTAN o NATO - habían rechazado ante unas definidas ofertas soviéticas. » (Revista SP, $\mathrm{n}^{\circ} 528$, page 51).

«Es lógico suponer que la presíon que ejerce esa capacidad sobrante sobre el mercado exterior permanezca por muchos años, aunque los proyectos de la NATO y alguna escasez debido a la huelga de estibadores de Estados Unidos. $\gg$ (Actualidad Economica, n 570, 15 février 1969, page 41).

Parfois on emploie UNO au lieu de ONU, mais il y a un peu d'ironie dans l'appellation :

«Es sabido que la UNO (ONU en castellano) fue beligerante en la guerra de 1939-1945. » (Revista SP, $\mathrm{n}^{\circ}$ 536, page 12).

D'autre part, certaines organisations internationales empruntent leur sigle à leur dénomination dans une des principales langues officielles, l'anglais ou le français. Ainsi GATT (General Agreement on Tariffs and Trade), IUPAC (International Union of Pure and Applicated Chemics), etc., qui s'emploie aussi bien en français qu'en espagnol, malgré «Accord Général sur les Tarifs et le Commerce » et « Acuerdo General sobre Aranceles y Comercio ».

De même pour le sigle UNESCO (United Nations Educational, Scientific and Cultural Organization) qui s'emploie également en français et en espagnol sous la même forme, mais il s'agit ici d'un sigle qui dans la pratique se substitue à une appellation en toutes lettres et que l'on peut considérer comme un acronyme.

\section{Identification}

L'identification du sigle pose un problème au traducteur. En dehors du cas particulier des sigles d'organisations internationales, il lui appartient de traduire soit par un sigle, soit par un terme ou une expression en toutes lettres, quel que soit le sigle rencontré dans un texte étranger, ou, s'il s'agit d'un sigle de valeur purement nationale, de le garder tel quel et d'indiquer la traduction la première fois qu'on le cite de sa dénomination en toutes lettres dans la langue d'origine de l'organisation ou de l'organisme qu'il représente.

Le traducteur doit donc être très prudent quand il utilise un sigle. Il ne peut le faire que s'il est très sûr de ses appellations et significations exactes et il ne pourra l'être qu'en consultant textes, périodiques, journaux..., et en se tenant au courant de toute création de nouvelles organisations.

\section{EMPLOI DES SIGLES}

Ces derniers temps on a développé, d'une manière exagérée peut-être, l'emploi des sigles pour abréger les noms, parfois trop longs, de pays, sociétés, institutions diverses, etc., jusqu’à former, dans certains cas, un ensemble prononçable. Aussi 
ne parle-t-on plus de 1 '《Union des républiques socialistes soviétiques », mais de l'URSS (et même Urss) ; de la RDA, et non plus de la « République démocratique allemande ».

Selon Larousse (Diccionario enciclopédico en tres volumenes, adaptation hispano-américaine du Nouveau Larousse universel dirigé par Claude et Paul Augé), il faut censurer cet emploi : "L'emploi abusif des sigles apporte parfois plus de confusion que de clarté, ce qui justifie la formation de glossaires $»$.

Les sigles sont d'usage courant dans :

1) Organisations internationales (ONU, UNESCO)

2) Partis et mouvements politiques (F.D.F., P.C.B., P.S.C.)

3) Sociétés commerciales (I.N.I.)

4) Marques (voir le Diario Oficial de El Salvador) :

G.S.D. George S. Dahbura

I.A. Israel Aguilar

J.A.Z. Juan Antonio Zablah

J.F.G. Juan Francisco Granados

J.I.A. José Israel Aguilar

5) Code abréviatif interbanquaire :

BDT «Bons du Trésor »

FDV Frais divers

MOR Mandat Ordonnancé

RBT Remboursement

6) Services télégraphiques et téléphoniques :

BUIT Bureau de l'Union Internationale des Télécommunications

PTT Postes, Télégraphes, Téléphones

SFR Société Française Radioélectrique

UIR Union Internationale de Radiodiffusion

UIT Union Internationale des Télécommunications

7) Service postal :
A.P.
Agence Postale
C.P.
Colis Postal
P.T.T.
Postes, Télégraphes, Téléphones
R.
Recommandé
U.P.U.
Union Postale Universelle
L.R.
Lettre Recommandée

8) Chèques postaux :

M.C. Mandat-Carte

T.L.M. Télégramme-Lettre-Mandat

T.V. Virement télégraphique international 
9) Métallurgie :

ARES «Aceros rápidos extra superiores》

ARO «Aceros rápidos ordinarios 》

ARS «Aceros rápidos superiores »

10) Notations techniques:

Ce sont plutôt des symboles. Ils s'écrivent sans point terminal :
A
C
air, arc
D angle droit, cinq cents (en chiffre romain), diamètre
capacité, cent (en chiffre romain)
G (ou d), droite (ligne).
gramme

11) Marques de voitures :

$\begin{array}{ll}\text { A } & \text { Autriche } \\ \text { B } & \text { Belgique } \\ \text { C } & \text { Cuba } \\ \text { CO } & \text { Colombie } \\ \text { E } & \text { Espagne } \\ \text { EQ } & \text { Equateur } \\ \text { G } & \text { Guatémala } \\ \text { MEX } & \text { Mexico } \\ \text { NA } & \text { Nicaragua } \\ \text { P } & \text { Paraguay } \\ \text { PA } & \text { Panama } \\ \text { PE } & \text { Pérou } \\ \text { RA } & \text { Argentine (République Argentine) } \\ \text { RD } & \text { République Dominicaine } \\ \text { U } & \text { Uruguay }\end{array}$

12) Biologie :

A.D.N. et A.R.N. : « Acide désoxyribonucléique » et « Acide ribonucléique ». Ce sont les deux formes d'acides nucléiques. On les trouve surtout dans les chromosomes au centre du noyau cellulaire. Ils forment le cerveau, l'ordinateur qui commande la fabrique cellulaire.

13) Physique :

$\begin{array}{ll}\text { A } & \text { Ampère } \\ \text { AL } & \text { Année-Lumière } \\ \text { J } & \text { Joule } \\ \text { MJ } & \text { Mégajoule... } \\ \text { O } & \text { Ohme } \\ \text { Q } & \text { Chaleur de réaction } \\ \text { V } & \text { Volt } \\ \text { W } & \text { Watt }\end{array}$


Onde TE: «Transverse Electric Wave» (Onde Electrique Transversale)

Onde TEM : «Transverse Electromagnetic Wave» (Onde Transversale Electro-magnétique)

Onde TM: «Transverse Magnetic Wave» (Onde Magnétique Transversale)...

14) Chimie :

Les symboles chimiques s'écrivent sans point terminal :

$\begin{array}{ll}\text { A } & \text { Argon } \\ \text { C } & \text { Carbone } \\ \text { F } & \text { Fluor... } \\ \text { H } & \text { Hydrogène } \\ \text { I } & \text { Iode } \\ \text { O } & \text { Oxygène... }\end{array}$

15) Musique :
D.C.
Da Capo

F.

FF. et F.F.F. Fortissimo

P. Piano

P.P. et P.P.P. Pianissimo

V.S. Volti subito...

16) Domaine de l'espionnage

Les divers services qui s'occupent des tâches de l'information aux ÉtatsUnis ont des sigles qui rappellent l'aridité de n'importe quelle société anonyme, bien qu'ils soient plus actifs que toutes les sociétés civiles du monde. C'est ainsi qu'une loi créa en 1947 la C.I.A. (Central Intelligence Agency : Agence Centrale de Service secret américain), dont le rôle dans l'histoire politique du monde contemporain allait être fréquemment important et, en certaines occasions, décisif. Ce n'est pas tout pour l'information secrète américaine. Le F.B.I. (Federal Bureau of Investigation : Bureau fédéral de Recherches) s'occupe, avec succès, des tâches de contre-espionnage et de sécurité intérieure, un peu comme le MI 5 britannique. Il existe encore la D.I.A., fusion de tous les services militaires d'information.

Dans cette mer universelle de l'espionnage nagent, avec les trois grands services (S.I.S., K.G.B., C.I.A.), les services secrets d'autres puissances : le B.N.D. de l'Allemagne de l'Ouest, par exemple.

Dans les dures années de Londres de 1940, de Gaulle avait ordonné la création d'un service secret qui prit le nom de B.C.R.A. (Bureau Central de Recherche et d'Action), qui fusionna en 1943 avec le D.S.S. (Direction des Services Spéciaux) sous les sigles de ce dernier. En 1945, le colonel Passy reçut l'ordre de réorganiser les services secrets. Il créa le SDECE, qui fut placé sous la direction et l'autorité du chef de l'État. 
En Italie, il y a le SIFAR, lié très fort aux forces armées.

a) Allemagne de l'Ouest

B.f.v. - Bundesverfassungsschutz. Service de protection constitutionnelle. C'est le service de protection de la Constitution. Sa création remonte à l'interdiction par la Constitution des partis politiques dirigés contre l'existence de l'État. Son activité se limite au territoire national pour lutter contre «toute action illégale qui pourrait menacer l'État ».

B.N.D. - Bundesnachrichtendienst. Service d'information fédéral. C'est le service le plus important d'Allemagne de l'Ouest. C'est l'officialisation du réseau « Gehlen ».

L'activité du B.N.D. s'exerce surtout sur le plan international. Il consiste à réunir des informations d'ordre militaire, politique, économique, et contrecarrer l'action des services adverses. Le passé du général Gehlen, qui avait dirigé pendant la guerre, sous l'autorité de l'amiral Canaris, l'espionnage dans les pays de l'Est (Fremde Heere Ost), lui assura son autorité dès la fin de la guerre. Grâce à son expérience et à ses spécialistes toujours disponibles, Gehlen devint un auxiliaire précieux pour le gouvernement américain. Ce ne fut qu'en 1955 que l'organisation fut placée sous l'autorité du gouvernement. Auparavant, elle était sous le contrôle direct du chancelier. Depuis lors, sa réputation ne cessa d'augmenter.

M.A.D. - Militaerischer Abschirmdienst. Service d'information militaire. Chargé de protéger les secrets de la Bundeswehr, elle veille au fonctionnement de l'armée.

b) Allemagne nazie

ABWEHR - Service d'Information Militaire de Hitler. Dirigé par le fameux amiral Canaris, il devint une force très bien entraînée et hautement efficace.

GESTAPO - Geheime Staatspolizei (Police secrète d'État).

KRIPO - Kriminalpolizei.

RSHA - Bureau de sécurité du Reich. C'était le centre de toute l'activité d'information du III ${ }^{e}$ Reich. Heinrich Himmler le dirigeait.

SCHUPO - Schutzpolizei. (Police de protection)

SIPO — Sicherheitspolizei. (Police de Sécurité)

VOPO - Volkspolizei. (Police populaire)

c) États-Unis

C.I.A. - Central Intelligence Agency. Agence centrale de renseignements. C'est le service secret le plus puissant des États-Unis. On le rencontre dans toutes les interventions, camouflées ou non, des Etats-Unis.

La C.I.A. est la plus connue des organisations chargées de recueillir des renseignements pour renforcer la sécurité des U.S.A., elle ne constitue que le dernier échelon d'une chaîne beaucoup moins connue : F.B.I., "Atomic Energy Commission 》, "Intelligence and Research Service », N.S.A., etc. 
C.I.C. - Council Intelligence Corps. C'était le service de renseignements militaires américains après la guerre.

DIA. - Defense Intelligence Agency. C'est le service de renseignements du Pentagone. Son siège se trouve à Arlington (Virginie), très près de Washington.

Il naquit à la suite de la fusion des services militaires de renseignements américains. Il dépend du Ministère de la Défense.

Pendant très longtemps, il fut en concurrence avec la C.I.A. Robert McNamara voulait libérer le plus possible l'administration américaine du monopole de fait que constituait la C.I.A. dans le domaine de l'information.

La D.I.A., dont les crédits et les effectifs (secrets) rivalisaient avec ceux de la C.I.A., coordonne les services d'informations des trois armes qui en vinrent aux mains avant 1961 : il s'agit de la Air Force Intelligence (qui se dédie à l'évaluation de la puissance aérienne et spatiale des pays étrangers de même qu'à la détermination des bombardements éventuels), du G-2 (service de renseignements de l'armée de terre) et de la Office of Naval Intelligence.

F.B.I. - Federal Bureau of Investigations. Responsable de la sécurité intérieure des États-Unis, ainsi que de l'espionnage et contre-espionnage sur le territoire américain.

N.S.A. - National Security Agency. Elle fut créée en 1952. Son but officiel est « la codification et la décodification des messages en provenance de tous les pays y compris les pays amis, c'est-à-dire qu'elle examine tous les messages émis par les stations étrangères (bases militaires, ambassades, navires, avions, etc.). Elle élabore aussi de nouveaux moyens techniques d'émission et d'interception.

Elle ne fait pas partie du Pentagone, mais elle communique avec la présidence par l'intermédiaire du Secrétaire de la Défense.

O.S.S. - Bureau des Services Stratégiques. Espèce de "père de la C.I.A. 》. Il centralisait les opérations spéciales pendant la guerre. Dulles dirigea la section en Suisse.

\section{d) France}

D.S.S. - Direction des services spéciaux. C'est l'embryon des services français actuels. Né à Alger pendant la guerre, il n'existe plus.

D.S.T. - Direction de la surveillance du territoire. C'est le contre-espionnage français. Il se trouve sous l'autorité du Ministre de l'Intérieur. Ses fonctionnaires sont des officiers de police judiciaire; leur tâche est de rechercher les agents de puissances étrangères qui opèrent en France. Quand ils accumulent les charges suffisantes, ils doivent déférer devant le Parquet ceux qu'ils estiment coupables et mettre à la disposition de la justice les actes qu'ils ont établis. Le juge de paix qui désire compléter son information leur confiera la tâche d'exécuter des commissions rogatoires.

S.D.E.C.E. - Service de documentation extérieur et de contre-espionnage. C'est le service secret français. Il se trouve sous l'autorité du Ministre des Armées 
et est l'héritier du Bureau central de renseignements et d'action (B.C.R.A.) de la France libre, puis Direction générale des études et recherches (D.G.E.R.)

Sa tâche est de collationner hors du territoire national toutes les informations et documentation susceptibles d'informer le gouvernement pour que soient signalés aux administrations intéressées les agents des puissances étrangères susceptibles de porter préjudice à la défense nationale ou à la sécurité de l'État.

e) Grande-Bretagne

D.G. - Voir M.I.5.

J.I.C. - Joint Intelligence Committee. Il centralise les activités de tous les services de renseignements.

Il se compose des chefs de chaque organisation de renseignements et de quelques représentants du gouvernement placé sous la présidence d'un haut fonctionnaire du Foreign Office.

M.1.5. - À l'origine Military Intelligence 5, plus connu sous les initiales «D.G. ». C'est la section du contre espionnage britannique du Service de sécurité. Son personnel se compose principalement d'inspecteurs de police et d'anciens fonctionnaires.

Sa fonction principale consiste au maintien à jour de plus de deux millions de dossiers en donnant des détails sur des personnes suspectes ou autres qui ont présenté de l'intérêt à un moment donné, ou qui auraient pu avoir de l'intérêt.

La mission du « DG » se limite surtout à la Grande-Bretagne, où il est responsable de la formation et de l'organisation du travail des agents qui doivent s'opposer aux activités d'espions étrangers.

M.I.6. - À l'origine Military Intelligence 6, il se dédie maintenant à l'espionnage actif. La faible sympathie qu'éprouvent l'un pour l'autre les deux services (M.I.5 et M.I.6) est célèbre.

S.I.S. - Secret Intelligence Service, ou M.I.6. C'est le légendaire Service secret de sa Majesté britannique. Il est chargé de la réunion de renseignements sur l'étranger. Bien qu'en théorie il chapeaute tout l'appareil de l'information secrète, on le confond généralement avec le M.I.6.

S.O.E. - Special Operation Executive. Il fut créé au début de la seconde guerre mondiale.

f) Italie

SIFAR - Service de renseignements des forces armée de la république.

g) Russie

G.R.U. - Direction principale de l'information de l'URSS. C'est l'organisme unique pour la recherche et l'exploitation de l'information. Il dépend complètement de l'armée.

Le chef du G.R.U. est un officier général de haut rang : général d'armée ou au moins colonel-général. Le G.R.U. est à la fois un bureau classique d'état-major et un centre de recherche de l'information. 
On suppose que l'organisme se subdivise en : cabinet du général, services administratifs et financiers, et quatre sections principales : l'ensemble des services techniques, l'exploitation (qui représente le cerveau du G.R.U.), la recherche (razvedka) et les centres de formation des cadres.

G.U.K.R. - Direction principale du contre-espionnage. C'est à la fois un organisme de détection, de prévention et de répression. Une partie importante de ses cadres se trouvent groupés au ministère de la défense où ils constituent une direction du contre-espionnage qui se ramifie au sein des forces armées.

Le G.U.K.R. a un réseau important d'informateurs par tout le pays.

K.G.B. - Comité de sécurité de l'État. C'est le tout-puissant service de renseignements russe. Il dépend officiellement du Conseil des ministres de l'URSS ; en réalité, il dépend du Politbureau, l'instance majeure du parti.

M.G.B. - Ministère de la sécurité de l'État. Il fut remplacé par le K.G.B. à la suite de la liquidation de Beria en 1953.

R.U. - Direction de l'information. C'était le « $4^{\mathrm{e}}$ Bureau » de l'État-Major général de l'armée rouge. Pendant la dernière guerre, il devint la « Direction principale de l'information » (G.R.U.) et prit le $\mathrm{n}^{\circ} 2$ au sein de l'État-Major général des forces armées soviétiques.

17) Sigles de l'ère spatiale

ATM - Apollo Telescope Mount. C'est une station astronomique. Ce devait être en réalité une S IV-8, étage de fusée qui a un diamètre de 6,57 mètres et fait partie des Saturn V.S IV-8 constitue le second et dernier étage d'une Saturn I-B ; c'est le troisième étage d'une Saturn V.

LINAC Accélérateur linéaire. de Chicago.

NAL - National Accelerator Laboratory. - On le trouve à Batavia, près

NASA - National Aeronautic and Space Agency.

R.M.N. - Résonnance magnétique nucléaire.

UFE-C. - Usine à faible enrichissement gros étages. Fabrique française d'uranium enrichi.

U.S.G. - Usine à super grosse. Fabrique française d'uranium enrichi.

U.T.G. - Usine très grosse.

18) Sigles de la terreur

Une série de sigles qui vont depuis une simple lettre jusqu'au nom spécifique d'un type déterminé de fusée ont fait que le monde ferme les yeux de peur en espérant que personne, en une circonstance historique déterminée, appuie sur un simple bouton après avoir reçu un ordre qui conduirait le monde à son autodestruction.

ABM - Antiballistic System. Ensemble de missiles antimissiles. En URSS on l'appelle le système GALOSH : il protège plusieurs villes soviétiques. Il se 
compose de deux types de missiles: le Galosh, avec une ou plusieurs charges nucléaires, et le Griffon, avec une portée de 250 kilomètres.

Aux USA, le programme anti-missiles Sentinelle destiné à protéger une vingtaine de grandes villes américaines a été remplacé par le projet Sauvegarde qui protège douze bases de lancement.

ICBM - Intercontinental Ballistic Missile. Engin ballistique américain à portée intercontinentale. Les États-Unis possèdent 1054 missiles de ce genre avec charge nucléaire qui varie de 10 à 100 mégatonnes. Portée : 12000 à 15000 kilomètres.

$I R B M$ - Intermediate Range Ballistic Missile. Engin ballistique américain, avec une portée de plus de 3000 kilomètres.

FOBS - Fractional Orbital Bombardment System. Programme soviétique qui consiste à mettre une bombe nucléaire en orbite poussée par un missile SS-9.

MIRV - Multiple Role Independently Tagetable Reentry Vehicle. Programme américain qui consiste à doter les missiles de plusieurs têtes nucléaires d'orientation variable. Le Minuteman-3 contient trois têtes et fut opérationnel à partir de 1971. Le Poséidon contient jusqu'à dix têtes. Trente et un des quarante et un submersibles atomiques américains doivent changer leurs missiles classiques par des projectiles de ce type.

MOL - Mobil Orbit Laboratory. Projet américain, pour le moment abandonné, destiné au montage d'un satellite nucléaire.

MRBM - Medium Range Ballistic Missile. Engin ballistique américain de portée moyenne de 3000 kilomètres.

PAR ET MSR - Perimeter Acquisition Radar et Missile Site Radar. Installation à terre de radars destinés à détecter les buts pour les missile Spartan et Sprint.

SAC - Strategic Air Command. Système américain composé d'une flotte de six cents bombardiers (B-52, B-58 et FB-III), avec une charge nucléaire, par unité de 13 à 24 mégatonnes. Rayon d'action illimité. Ravitaillement en vol.

SLCM - Submarine-Lauched Cruise Missile. Les Américains disposent de quarante et un submersibles nucléaires armés chacun de 16 missiles Polaris avec une charge supérieure au mégatonne. Sa portée est de 2500 à 4800 kilomètres.

19) Sigles généraux
A.
Altesse
A.I.
Altesse Impériale
A.M.
Ante meridiem
A.R.
Altesse Royale
A.C.
Année du Christ
B.L.M.
«Besa la mano »
B.L.P.
«Besa los pies »
C.P.B.
«Cuyos pies beso»
F.
«Fulano »
N.
« Fulano, persona cuyo nombre se ignora $» ;$ «norte » 


$\begin{array}{ll}\text { N.B. } & \text { Nota bene } \\ \text { N.S.J.C. } & \text { Notre Seigneur Jésus-Christ } \\ \text { O. } & \text { Ouest } \\ \text { P.M. } & \text { Post meridiem } \\ \text { Q.B.S.M. } & \text { «Que besa su mano » } \\ \text { Q.B.S.P. } & \text { «Que besa sus pies » } \\ \text { Q.D.G. } & \text { «Que Dios guarde » } \\ \text { R.I.P. } & \text { Requiescat in pace } \\ \text { S.A.R. } & \text { Son Altesse Royale } \\ \text { S.C.M. } & \text { «Su Catolica Majestad » } \\ \text { S.D.M. } & \text { Sa Divine Majesté } \\ \text { S.M. } & \text { Sa Majesté } \\ \text { S.M.C. } & \text { « Su Majestad Catolica » } \\ \text { V.M. } & \text { Votre Majesté ; «Vuestra Merced }\end{array}$

\section{PETITE BIBLIOGRAPHIE SUR L'ÉTUDE DU SIGLE}

En allemand

1. Abkürzungen, Abréviations, Abbreviations, Swiss Bank Corporation, London, 1964.

2. BRENNIGER, A., "Italienische Abkürzungen », Lebende Sprachen, $\mathrm{n}^{\circ} 5$, septembreoctobre 1971, p. 146.

3. BRUNOLD, Hans Peter, "Englishsprachlige Abkürzungen", Lebende Sprachen, $\mathrm{n}^{\circ} 5$, septembre-octobre 1971, p. 135 et 136.

4. CAPELLI, Adriano, Wörterbuch lateinischer und italienischer Abkürzungen, wie sie in Urkunden und Handschriften, besonders des Mittelalters, gebrailchlich sind, dargestellt in über 14.000 Holzschnittzeichen, $2^{\circ}$ verbesserte Auflage, Leipzid, J. J. Waber, 1928, $16^{\circ}$, p. II, LXVI-543 p. (J. J. Webers illustrierte Handbücher).

5. DES COUDRES, Hans Peter, Juridistische Abkürzungsverzeichnisse, Berlin, Walter de Gruyter; Tübingen, J.C. B. Mohr, $1954,8^{\circ}, 26$ p. (Sonderdruck aus Zeitschrift für ausländisches und internationales Privatrecht. xvin, 2, p. 524-549).

6. DIETRICH, Felix, Internationale Bibliographie der Zeitschriftenliteratur aus allen Gebieten des Wissens, Felix Dietrich Verlang, Osnabruick, 1968, 1969, 1970 et 1971.

7. GOETTLING, Hans, voir SPILLNER.

8. GREISER, Josef, Lexikon der Abkürzungen. Mit Anhang : Abkürzungen aus dem Finanzund Steuerrecht, Osnabrïck, A. Fromm, 1953, 12 $231 \mathrm{p}$.

9. GREISER, Josef, Lexikon der Abkürzungen. Mit Anhang : Abkürzungen aus dem Finanzund Steuerrecht. 2. Auflage ergänzt und erweitert nach dem neuen Stande vorn I. Januar Osnabrück, A. Fromm, 1955, 12, 271 p.

10. KOBLISHCHKE, Heinz, Abkïrzungsbuch (Abkürzungen - Kurzwörter - Zeichen Symbole), Veb Bibliographisches Institut, Leipzig, 1969, $8^{\circ}$, ill., 369 p.

11. LANZE, Werner, DABI - Das Abkürzungsbuch für den Ingenieur (Sammlung von Fachausdrücken aus dem Gebiet der Technik sowie aus den angrenzenden Bereichen der Wirtschaft, der Politik und der entsprechenden Organisationen), Vulkan-Verlag Dr. W. Classen, Essen, 1969.

12. PFOHY, Ernst, Kurzwort - Lexikon, K.W.L., Stuttgart, Muth, 1934, $8^{\circ}$, XII - 394 p.

13. ROTHE, H., Akülex. Taschenlexikon der Abkürzungen, Frankfurt/M., 1956.

14. RUPPERT, Fritz, Initials - Abkürzungen von Namen internationaler Organisationen, Verlag Dokumentation, München-Pullag, Gemeinschaftsauflage mit dem Vulkan-Verlag Dr. W. Classsen, Essen, $1966,18^{\circ}, 220$ p. 
15. SANGHOLLE, Marie-Carmen, "Französische Abkürzungen ", Lebende Sprachen, $\mathrm{n}^{\circ}$ 5, septembre-octobre 1971, p. 141.

16. SAUR, Karl-Otto, "Handbuch der Technischen Dokumentation und Bibliographie" (Internationales Verzeichnis von Abkürzungen con Verbänden, Behörden und Organisationem), Bearbeitet von Karl-OttoSaur und Grete Gringmuth, Teil I und 2, Verlang Dokumentation, München-Pullach, 1968.

17. SCHEITZ, Edgar, Russische Abkürzungen und Kurzwörter (russich-Deutsch), Berlin, Verlag * Technick», 1961, 8, xv $-727 \mathrm{p}$.

18. SPILlNER, Paul \& GOETTLING, Hans, Buch der Abkürzungen, Bamberg, C. C. Buchners Verlang, $1952,8^{\circ}$, vII $-159 \mathrm{p}$.

19. TARNOCZI, Lorant, Zur Frage der Abkürzungszeichen in Zeitschrift für Phonetik Sprachwissenschaft und Kommunikationsforschung, p. 272-284, Akademie, Verlang, Berlin, 1969.

20. TRAUBE, Ludwig, Versuch einer Geschichte der christlichen Kürzung, München, C. $\mathrm{H}$. Beck, $1907,8^{\circ}$, portr., $\mathrm{X}-295$ p. (Quellen und Untersuchungen zur lateinischen Philologie des Mittelalters, 2).

21. VOLLMER, Friedrich, Kürzung durch Tonanschluss in alten Latein, München, J. Roth, $1917,8^{\circ}, 32$ p. (Sitzungsberichte der Königlich Bayerischen Akademie der Wissenschaften. Philosophisch - philologische und Historische Klasse ; 1917, 9).

En anglais

22. Acronyms Dictionary, Detroit, 1960.

23. BAKO, Elemer, Hungarian Abbreviations. A Selective List, Library of Congress, Reference Department, Slavic and Central European Division, Washington, 1961, $8^{\circ}$, IV $146 \mathrm{p}$.

24. BASTINGS, Ed. L., Directory of New Zealand Science, Wellington, 1954 ( $3^{\mathrm{rl}}$ Edition).

25. BUTTRESS, Frederick Arthur, World List of Abbreviations (over 9000 items), Leonard Hill, London, 1966 ( $^{\text {rd }}$ Edition), $8^{\circ}$, vI $-186 \mathrm{p}$.

26. COLLINS, Frederick Howard, Authors' and Printers' Dictionary, A guide for authors, editors, printers, correctors of the press, compositors, and typists. With full list of abbreviations. An attempt to codify the best typographical practices of the present day. London, Humphrey Milford, 1928 ( $6^{\text {th }}$ Edition $-1^{\text {st }}$ Edition : May 1905), $16^{\circ}, x v-$ $406 \mathrm{p}$.

27. COLLINS, Frederick Howard, Authors and Printers' Dictionary. A guide for authors, editors, printers, correctors of the press, compositors, and typists. With full list of abbreviations. An attempt to codify the best typographical practices of the present day. London, Humphrey Milford, 1933 ( $7^{\text {th }}$ Edition, $8^{\text {th }}$ impresssion revised), $8^{\circ}$, XVI $407 \mathrm{p}$.

28. COLLINS, Frederick Howard, Authors' and Printers Dictionary. A guide for authors, editors, printers, correctors of the press, compositors, and typists. London - New York - Toronto, Geoffrey Cumberlege, 1956 (10 ${ }^{\text {th }}$ Edition $), 18^{\circ}$, XvI $-442 \mathrm{p}$.

29. COLlins, J., Acronyms, Part V of the "Directory of Scientific Resources in South Africa ", Pretoria, 1963 (2 $2^{\text {nd }}$ Edition).

30. COPE, S.T., Glossary of Abbreviations with particular reference to the Telecommunications Industry, Marconi Co., Chelmsford, 1955.

31. COPE, S. T., Glossary of Abbreviations for Names of Technical, Scientific, Industrial and Professional Organizations, Chelmsford, 1957.

32. CROWLEY, Ellen T. \& THOMAS, Robert C., Acronyms and Initials Dictionary (about 45000 entries), (A Guide to Alphabetic Designations, Contractions, Acronyms, Initialisms, and Similar Condensed Appellations Covering Aerospace, Associations, Biochemistry, Business and Trade, Domestic and International Affairs, Education, Electronics, Genetics, Government, Labor, Medicine, Military, Pharmacy, Psysiology, Politics, Religion, Science, Societies, Sports, Technical Drawings and Specifications, Transportation, and Other Fields), Gale Research Company, Book Tower, Detroit/Michigan, 1970 ( $3^{\text {rd }}$ Edition). (To be supplemented by : New Acronyms and Initialisms, 1971: New Acronyms and Initialisms, 1972). 
33. CROWLEY, Ellen T., Reverse Acronyms and Initialisms Dictionary, A companion volume to acronyms and initialism dictionary, with terms arranged alphabetically by meaning of acronyms. Edited by Ellen T. Crowley \& Robert C. Thomas (with Contributors), Detroit, Gale, $1972,4^{\circ}, x-485 \mathrm{p}$.

34. —_ Directory of Scientific Institutes, Organizations and Services, Scientific Council for Africa South of the Sahara, Publ. $n^{\circ} 14$, London, 1954.

35. —_- Encyclopedia of Associations - National Organisations of the United States, Gales Research Co., Detroit, 1961 ( $3^{\text {ra }}$ Edition).

36. - English and American Abbreviations Dictionary (31 000 entries), Moscow, 1957.

37. FAWCETT, Frank Dubrez, Cyclopaedia of Initials and Abbreviations, Business Publications, London, $1963,8^{\circ}$, VIII $-185 \mathrm{p}$.

38. - Federation of British Industries Register of British Manufacturers, London (Annuel).

39. FURNESS, Konstantin Z., Bulgarian Abbreviations. A Selective List, Library of Congress, Reference Department, Slavic and Central European Division, Washington, 1961, $4^{\circ}$, $326 \mathrm{p}$.

40. - Glossary of Abbreviations Relating to African Affairs, Research and Microfilm Publications (U.S. Government translation), CCM Information Corporation, 1963.

41. - Glossary of Symbole and Abbreviations (Bilingual : English/French), O.E.E.C., Paris, 1956 (and H.M.S.O., London).

42. GOLDSTEIN, M., Dictionary of Modern Acronyms and Abbreviations, Bobbs-Merrill Co., Indianapolis, New York, 1963.

43. GURNETT, John William \& KYTE, C.H.J., Cassell's Dictionary of Abbreviations (over 21000 entries), Cassel \& Company Ltd., London, 1966, 16 ${ }^{\circ}$, vil $-220 \mathrm{p}$.

44. HORECKY, Paul L., Czech and Slovak Abbreviations. A Selective List. Edited by Paul L. Horecky, Washington, Library of Congress, Slavic and Central European Division, 1956, $4^{\circ}, \mathrm{v}-164 \mathrm{p}$.

45. Industrial Research Laboratories of the United States, Publ. $\mathrm{n}^{\circ}$ 884, National Research Council, Washington, 1961 (II ${ }^{\text {th }}$ Edition).

46. - International Initialese, Publ. $\mathrm{n}^{\circ}$ 182, Union of International Associations, Brussels, 1963 ( $^{\text {nd }}$ Edition).

47. —_- International Scientific Organisations, Library of Congress, Washington, 1962.

48. KORITSKII, B. F., Dictionary of Abbreviations of the Russian Language, (Russian text), State Publishing House, Moscow, 1963.

49. KYTE, C. H. J., voir GURNETT, J. W.

50. MATTHEWS, C.C., A Dictionary of Abbreviations, London, 1947.

51. MILLARD, P., Trade Associations and Professional Bodies of the United Kingdom, Lechworth, 1964 ( $2^{\text {nd }}$ Edition).

52. MOSER, Reta C., Space-Age Acronyms. Abbreviations and Designations (over 15000 entries), IFI/PLENUN, Data Corporation Plenun Publishing Company Ltd,, New York/ Washington/London, 1969 ( $2^{\text {nd }}$ Edition Revised and Enlarged).

53. PATRICK, G. Z., A List of Abbreviations Commonly Used in the U.S.S.R., University of California, Berkeley, 1937.

54. PARTRIDGE, Eric, A Dictionary of Abbreviations, With Special Attention to War-Time Abbreviations, London, Allen \& Unwin, $1943,8^{\circ}, 105$ p.

55. PLAMENATZ, Ilija J., Yugoslav Abbreviations. A Selective List. Washington, Library of Congress, Reference Department, Slavic and Central European Division, 1959, $4^{\circ}$, $\mathrm{v}-185 \mathrm{p}$.

56. PUGH, Erich, A Dictionary of Acronyms and Abbreviations. ( + a comprehensive subject index) (over 10,000 entries), some abbreviations in management, technology and information science), Archon Books \& Bingley, London, 1970 (Second, Revised and Expanded Edition, $8^{\circ}, 389 \mathrm{p}$. 
57. —, Quarterly Bulletin, International Association of Agricultural Librarians and Documentalists (TAALD).

58. RAND, Edward Kennard, A Nest of Ancient Notae (with 6 plates), Cambridge (Mass.), The Mediaeval Academy of America, 1927, $4^{\circ}$, facsim., 17 p. (Offprinted from Speculum, II, 2 p. 160-176).

59. ROSENBERG, Alexander, Russian Abbreviations, A Selective List, Washington, Library of Congress, Slavic and Central European Division, 1952, 4.

60. ROSENBERG, Alexander, Russian Abbreviations. A Selective List, Washington, Library of Congress, Slavic and Central European Division, $1957\left(2^{\text {nd }}\right.$, Revised and Expanded Edition), $4^{\circ}, \mathrm{IX}-513 \mathrm{p}$.

61. ROSENBERG, Alexander, Russian Abbreviations. A Selective List, Washington, Library of Congress, Slavic and Central European Division, 1957, $\left(2^{\text {nd }}\right.$, Revised and Expanded Edition), $4^{\circ}, \mathrm{X}-513 \mathrm{p}$.

52. SCHWARTZ, Robert J., The Complete Dictionary of Abbreviations, London - Toronto - Wellington - Sydney, G. G. Harrap \& $\mathrm{C}^{\circ}, 1955,8^{\circ}, 211$ p.

63. SCHWARTZ, Robert J., The Complete Dictionary of Abbreviations, New York, Thomas Y. Crowell Cy, $1955,8^{\circ}, 211 \mathrm{p}$.

64. - Scientific and Learned Societies of Great Britain, Allen \& Unwin, London, 1964

65. - Scientific and Technical Societies of the United States and Canada, Publ. $\mathrm{n}^{\circ} 900$, National Research Council, Washington, 1961 ( $7^{\text {th }}$ Edition).

66. SOLA, Ralph de, Abbreviations Dictionary, Constable, London, 1969.

67. STEEN, Edwin Benzel, Dictionary of Abbreviations in Medicine and the Related Sciences, Philadelphia, 1960

68. STEEN, Edwin Benzel, Acronyms and Initialisms Dictionary, A guide to alphabetic designations, contractions, acronyms, intialisms and similar condensed appellations. Edited

by Robert $C$. Thomas. Contributing Editors : Edwin B. Steen. Detroit, Mich.; Gale Research $\mathrm{C}^{\circ}$, Book Tower ; 1965 ( $2^{\text {nd }}$ Edition ), $8^{\circ}, 767 \mathrm{p}$.

69. - Technical Services for Industry, Department of Scientific and industrial Research, London, 1965.

70. —, Whitakers Almanach, London (Annual).

71. WILKES, I., British Initials and Abbreviations, Leonard Hill, London, 1966 ( $2^{\text {nd }}$ Edition).

72. WOJCICKA, Janina, Polish Abbreviations. A Selective List, Washington, Library of Congress, Slavic and Central European Division, $1957\left(2^{\text {nd }}\right.$, Revised and Enlarged Edition ), $4^{\circ}$, IV - $164 \mathrm{p}$.

73. — World List of Abbreviations of Scientific, Technological and Commercial Organizations, London, 1960.

74. — World of Learning, Europa Publications, London (latest Edition).

75. —_ Y Yearbook of International Organizations, Union of International Organizations, Brussels, 1964/5 (10 Editions).

En français

76. - Abréviations de sociétés, conventionnelles et ustuelles, publié par le Lloyd anversois, Anvers, 14, rue Vleminckx, $1926,16^{\circ}, 64 \mathrm{p}$.

77. - Abréviations usuelles ( $3^{\circ}$ éd.) - Gebruikelijkse Afkortingen ( $3^{\circ}$ uitg.), Bruxelles, Sénat de Belgique, 1954, $8^{\circ}, 106 \mathrm{f}$. (Service d'études et de documentation du Sénat de Belgique - Studie - en documentatie dienst van de Senaat van België).

78. - Abréviations usuelles ( $4^{\circ}$ éd.) - Gebruikelijkse Afkortingen (4 $4^{\circ}$ uitg.), Bruxelles, Sénat de Belgique, 1956, $12^{\circ}$, (I) $-149 \mathrm{p}$. (Service d'études et de documentation du Sénat de Belgique - Studie - en documentatie dienst van de Senaat van België). 
79. —— Abréviations usuelles ( $5^{\circ}$ éd.) —-Gebruikelijkse Afkortingen ( $5^{\circ}$ uitg.), Bruxelles, Sénat de Belgique, $1958,4^{\circ}, 87 \mathrm{f}$. (Service d'études et de documentation du Sénat de Belgique - Studie - en documentatie dienst van de Senaat van België).

80. BAUDRY, Hubert, D.A., Dictionnaire d'abréviations françaises et étrangères, techniques et usuelles, anciennes et nouvelles, 8000 abréviations, la Chapelle-Montligeon (Orne), Editions de Montligeon, 1951, $8^{\circ}, 157$ p. (Les Collections de Montligeon. Section "Documents ", 17).

81. BAUDRY, Hubert, Nouveau dictionnaire d'abréviations. D.A. Dictionnaire d'abréviations françaises et étrangères, techniques et usuelles, anciennes et nouvelles, La ChapelleMontligeon (Orne), Editions de Montligeon, 1956, 24, $418 \mathrm{p}$.

82. BEVEL, Maurice-Louis, Quels sont ces sigles?, Editions M. L. Bevel, Bruxelles.

83. — Dictionnaire des sigles et abréviations dans les domaines de la construction, de la sociologie, de l'urbanisme, Centre scientifique de technique du bâtiment, Paris, décembre 1968 (Etabli par la. "Section Documentation du C.S.T.B. " sous la direction de J. Flitz, D. Raymal et M. Dorster, avec l'aide du "Groupe Urbanisme et Habitat de l'A.D.B.S. ").

84. - Dictionnaire des sigles et abréviations dans les domaines de la construction, de la sociologie, de l'urbanisme, Centre scientifique et technique du bâtiment, Paris, $1^{\mathrm{er}}$ additif à l'édition de décembre 1968.

85. - Liste provisoire d'abréviations, Bruxelles, Comité national Belge de la F.A.C., $1947,4^{\circ}, 7 \mathrm{f}$.

86. MAGUERIE, Jean de, Abréviations et sigles économiques et sociaux, Paris, Institut d'observation économique, 1955, gr. $8^{\circ}, 40$ p., dans "L'observation économique ", juillet-août 1955 .

87. MAGUERIE, Jean de, Abréviations et sigles économiques et sociaux, Paris, Publications économiques et sociales, 1959, 2 vol. $4^{\circ}, 32$ p. dans "Les Cahiers économiques" l'Observation économique, sociale et financière, $\mathrm{n}^{\circ} 139$ et 140 .

88. - Sigles - Abréviations, dans " Problèmes africains et du Tiers-Monde ", $\mathrm{n}^{\circ} 494$ à 505, mai-juillet 1969 .

89. __ Sigles agricoles. Organisations d'Intérêt Agricole à Cadre National. Répertoire Alphabétique, Assemblée Permanente des Présidents des Chambres d'Agriculture, Paris, 1965.

90. SCHUERMANS, M. H., Sigles figulins (Epoque romaine), Bruxelles, Librairie Européenne de C. Muquardt, $1867,8^{\circ}, 292$ p. (Extrait des Annales de l'Académie d'archéologie, tome XXIII, $2^{\mathrm{e}}$ série, tome III).

91. ZUMTHOR, Paul, Abréviations composées, Amsterdam, North-Holland Publishing Company, 1951, gr. $8^{\circ}, 75$ p., dans "Verhandelingen der Koninklijke Nederlandse Akademie van Wetenschappen. Afđeling Letterkunde. Nieuwe reeks, LVII, 2 ».

En italien

92. BATTELLI, Giulio, Lezioni di Paleografia, Città del Vaticano, Pont. Scuola Vaticana di paleografia e diplomatica, 1949 (3a edizione), $8^{\circ}, \mathrm{X}-274 \mathrm{p}$.

93. CAPPELLI, Adriano, Dizionario di abbreviature latine ed italione, Milano, Ulrico Heopli, 1949 (4a edizione - anastatica - corredate con 9 tabole fuori testo), $18^{\circ}$, facsim., 531 p. (Manuali Hoepli. Lexicon abbreviaturarum).

94. CAPPELLI, Adriano, Dizionario di abbréviature latine ed italiane, (usate nelle carte e codici specialmente del medio-evo riprodotte con oltre 14000 segni incisi con l'aggiuta di uno studio suela brachigrafia medio-evale, un prontuario di "Sigle Epigrafische », l'antica numerazione romana ed arabica ed i segni indicanti monete, pesi, misure, etc.), Editore Ulrico Hoepli, Milano (1954 5a edizione - anastatica - corredate con 9 tavole fuori testo).

95. TRAMONTI, Nino, Dizionario delle sigle e delle abbreviazioni, Busto Arsizio, Unione Bustese degli Industriali - UBI, 1958 (2a edizione) $8^{\circ}, 330 \mathrm{p}$. 
En latin

96. HULAKOVSKY, Joannes M., Abbreviaturae vocabulorum, usitatae in scripturis praecipue latinis medii aevi, tum etiam slavicis et germanicis, collectae et..., Pragae, M. A. Vitek, $1852,8^{\circ}, 78 \mathrm{p}$.

97. —_, Modus legendi abbreviaturas, passim in utroque iure occurentes, nunc demum integritati suae restitutus, Parisiis, Ex officina Claudii Chevallonii, $1526,8^{\circ}, 46 \mathrm{f}$.

98. — Modus legendi abbreviaturas passim in utroque jure occurentes, Parisiis, Chevallon, 1531.

99. NICOLAUS, Johannes, Tractatus de siglis veterum omnibus elegantioris literaturae amatoribus utilissimus, in quo continentur quae ad interpretationem numismatum, inscriptionum, juris et fere omnium artium requiruntur, cujus subsidio facile literae explicari possunt, Lugduni Batavorum, de Swart, 1703, $8^{\circ}, 314 \mathrm{p}$.

En néerlandais

100. JANSSEN, Dries, Van aab tot zoo, letterwoorden-boek Uztgeverij Heideland, Vlaamse poekets, $n^{\circ} 137$, Hasselt, 1964.

101. - Landbouwgids, Utrecht (Annual) (For the Netherlands).

102. KEESING, I. G., V.A.R.L.A. : Verklarend alfabetisch register van initialen en afkortingen, verzameld, gerangsckikt en toesgelicht, Herziene en sterk vermeerdele 2 de uitgave, Deventer, Kluwer, $1937,12^{\circ}, 138 \mathrm{p}$.

103. — Tuinbouwgids, Den Haag (Annual) (For the Netherlands).

En russe

104. SPISSOK, Rousskikh sokrachtchenii primenjamykk v SSSR, München, Institout po Izoutcheniou Istorii $\mathrm{i}$ Koultoury SSSR, 1954, $8^{\circ}, 304 \mathrm{p}$. (Institout po Izoutcheniou Istorii i Koultoury SSSR. Issledovaniie i materialy, seria $\mathrm{I}, \mathrm{n}^{\circ} 13$ ).

Multilingues

105. - Abbreviations (Dutch, English, French, German), Van Goor Zonen, Den Haag/ Brussel, 1969.

106. BLUWSTEIN, W., Dictionary of Abbreviations (in English, German, Dutch and Scandinavian Languages) over 33000 entries), "Soviet Encyclopaedia» Publishing House, Moscow, 1964.

Daniel Gehenot 